\title{
Spatial Firing Patterns of Hippocampal Complex-Spike Cells in a Fixed Environment
}

\author{
Robert U. Muller, John L. Kubie, and James B. Ranck, Jr. \\ Departments of Physiology and Anatomy and Cell Biology, Downstate Medical Center (SUNY), Brooklyn, New York 11203
}

\begin{abstract}
A TV/computer technique was used to simultaneously track a rat's position in a simple apparatus and record the firing of single hippocampal complex-spike neurons. The primary finding is that many of these neurons behave as "place cells," as first described by O'Keefe and Dostrovsky (1971) and O'Keefe (1976). Each place cell fires rapidly only when the rat is in a delimited portion of the apparatus (the cell's "firing field"). In agreement with O'Keefe (1976) and many other authors, we have seen that the firing of place cells is highly correlated with the animal's position and is remarkably independent of other aspects of the animal's behavioral state.
\end{abstract}

Several properties of firing fields were characterized. Firing fields are stable over long time intervals (days) if the environment is constant. They come in several shapes when the animal is in a cylindrical apparatus; moreover, the set of field shapes is different when the animal is in a rectangular apparatus. It also seems that a single cell may have more than one field in a given apparatus. By collecting a sample of $\mathbf{4 0}$ place cells in a fixed environment, it has been possible to describe certain features of the place cell population, including the spatial distribution of fields within the apparatus, the average size of fields, and the "intensity" of fields (as measured by maximum firing rate). We also tested the hypothesis that the firing rate of each place cell signals the animal's distance from a point (the field center) so that a weighted average of the firing of the individual cells encodes the animal's position within the apparatus. The animal's position, calculated according to this "distance hypothesis," is systematically different from the animal's true position; this implies that the hypothesis in its simplest form is wrong.

The hippocampal place cells discovered by O'Keefe and Dostrovsky (1971) and more fully characterized by O'Keefe (1976) are named for their property of firing rapidly only when a freely moving rat is in a limited region of the space accessible to the animal; this region will be called the cell's "firing field." As a result of the large difference between in-field and out-of-field

\footnotetext{
Received Mar. 17, 1986; revised Dec. 1, 1986; accepted Jan. 8, 1987.

This work was supported by NIH Grants NS 20686 to J.L.K. and R.U.M and NS 14497 to J.B.R., Jr. We are indebted to Mr. Bobby Marsh of the Department of Physiology for designing and building the interface that allows the animal's position to be tracked and encoded as a binary number that can be sent to a computer. We thank Dr. Elizabeth Bostock for experimental contributions, Dr. Steven E. Fox for much help and encouragement, and Drs. John O'Keefe and David Zipser for many useful discussions.

Correspondence should be addressed to Dr. Robert Muller, Department of Physiology - Box 31, Downstate Medical Center, 450 Clarkson Avenue, Brooklyn, NY 11203.

Copyright (c) 1987 Society for Neuroscience $0270-6474 / 87 / 071935-16 \$ 02.00 / 0$
}

firing, simply watching a rat's position while monitoring the spikes generated by a cell is sufficient to get a good first impression of the location of a field. For this reason, the authors of most previous studies of place cells have been justified in using their own impressions of the existence and location of firing fields. Unfortunately, a good deal of this work has been less than convincing to individuals who have not directly seen place cell recordings. The first purpose of the work presented here was therefore to document the place cell phenomenon, using automatic means for locating the animal and simultaneously recording the firing of a place cell. The vidco-based method located the rat 60 times/sec in a $64 \times 64$ grid of small rectangular picture elements (pixels) of the video field, so that the raw data consisted of a time series of locations and a parallel time series of action potentials. Video/computer methods for locating the animal have been previously employed by O'Keefe (1983) and McNaughton et al. (1983a).

The location and spike series were used to generate timeaveraged, spatial distributions for the cell's firing rate by dividing the total number of spikes fired in each pixel by the total time the rat spent in that pixel. The spatial firing distributions can be inspected for the presence of firing fields by transforming them into color-coded maps of firing rate as a function of position. Firing rate maps are useful for determining, for example, the number of firing fields for a cell or for describing field shapes. A natural extension of documenting the existence of firing fields is characterizing some of their properties. Numerical values for other field properties, such as area or location within the apparatus, can be directly derived from the spatial firing rate. These methods will be used to describe average field properties for the place cell population.

Most individuals who "listen" to a place cell while observing a rat agree that firing does not occur in association with any spccial activity on the rat's part. Best and Ranck (1982) made this point by showing that naive viewers of videotapes with spike data on the sound track chose location as the best correlate of firing. A second purpose of the present work was to measure place cell firing in a reduced behavioral situation, in order to reinforce the idea that place cell firing is "location-specific" rather than "behavior-specific." The optimal activity pattern would be for the rat to move constantly at the same speed, and to visit all parts of the recording chamber equally often, as would happen with a random walk. Because this behavior pattern is time-invariant, spatial inhomogeneities of firing would have to be due to the rat's location per se, and could not be ascribed to what the animal does while at a location. To approximate this ideal, rats were trained to recover small food pellets thrown into the recording chamber. Since the pellets arrive frequently, the animal spends much of its time chasing them and its behavior 
is reasonably homogeneous in time. Since the pellets scatter widely, the animal moves around the entire floor area, and its bchavior is also reasonably homogeneous in space. The rat visits all parts of the apparatus in the course of a recording session, so that the firing rate everywhere in the accessible space can be measured. If firing fields were found under these circumstances, it would be hard to argue that firing is due to particular behaviors expressed in particular locations. The task has the additional advantage that it need not be changed when the environment is altered, so that the contribution of the environment can be assessed while behavior is held constant (Muller and Kubie, 1987).

If place cell firing is in fact location-specific, it follows that sensory information is necessary for the firing to reliably occur at certain places and not others. The third major purpose of this work was, therefore, to ascertain the nature of stimulus control over place cell firing. This required a simple recording chamber whose major features could be identified and independently varied. The specifics of the recording chamber were selected according to additional considerations. First, it seemed best to use an apparatus that contained a region of unobstructed space much larger than the animal; in this case, the rat's motions were not always affected by the apparatus boundary. In addition to size, the floor plan was important, since it is possible that interesting effects of the boundary shape could be detected in the shapes of firing ficlds. A circular profile seemed reasonable for studying field shapes because of its simplicity and because it minimizes perimeter relative to area. The animal was confined in a cylinder rather than on a raised disk because the resulting area was independent of the rat's propensity to put its head over the edge of a disk, and because the physical and visual boundaries of a cylinder are very similar. The inner surface of the cylinder was gray, except for a single stimulus (a white cardboard sheet) attached to the wall. This "cue card" polarized the cylinder from the experimenters' viewpoint and was a potential determinant of the angular position of firing fields in the laboratory frame. Finally, the floor of the cylinder was made of gray paper. This design allowed the 3 main components of the apparatus - the wall, the cue card, and the floor area-to be individually manipulated.

This paper contains a description of the spatial firing properties of place cells recorded when the rat is moving around in the cylindrical apparatus. In the accompanying paper (Muller and Kubie, 1987), the spatial firing patterns of individual cells will be compared before and after the major components of the cylinder are modified. Together, these studies are an attempt to quantify the place cell phenomenon under very simple behavioral circumstances, and to lay a groundwork for future investigations.

A preliminary version of some of the work in this paper was given in abstract form in Muller et al. (1983) and Kubie et al. (1983).

\section{Materials and Methods}

\section{Apparatus and behavioral training}

The basic recording chamber is a cylinder $76 \mathrm{~cm}$ in diameter and 51 $\mathrm{cm}$ high. Its wall is made of plywood painted gray. The bottomless cylinder is placed on a piece of gray photographic backdrop paper that is easily renewed. On the wall during all training sessions and most recording sessions is a sheet of white cardboard, bent to conform to the inner surface. This "polarizing stimulus" is the only intentionally introduced asymmetry in the environment. It occupies $100^{\circ}$ of arc and extends from the floor to the top of the cylinder's wall. In all experiments in this paper, the polarizing stimulus is centered on $30^{\circ} \mathrm{clock}$, ds viewed from the overhead TV camera.

In addition to the small cylinder, 3 other recording chambers are available: The large cylinder $(152 \mathrm{~cm}$ diameter, $102 \mathrm{~cm}$ high) is scaled up by a factor of 2 from the small cylinder. The other 2 apparatuses have rectangular floor plans. The small rectangle is $48 \times 56 \mathrm{~cm}$ and 51 $\mathrm{cm}$ high. The large rectangle is scaled up from its smaller counterpart by a factor of 2 in all dimensions. Each of these chambers is painted gray and is fitted with a white card. For the large cylinder, the card occupies $100^{\circ}$ of arc and runs from floor to top; it is centered at 3 o'clock. For the rectangles, the card covers the short wall that is tangent to 3 o'clock. These 3 apparatuses were mainly used in the work reported in the following paper (Muller and Kubie, 1987).

The essential behavioral requirement for our experiments is that the animal visit all parts of the apparatus. Rats are induced to do this by training them to chase small food pellets. During all phases of training and testing, young female hooded rats are food-deprived for $22 \mathrm{hr} / \mathrm{d}$, 5-7 d/week. On a rat's first training day, it is put in the small cylinder after approximately fifty $45 \mathrm{mg}$ food pellets have been scattered on the floor. It is left in the apparatus for $30 \mathrm{~min}$, during which most rats move around and eat the food. On subsequent training days, each rat is given four 15 min sessions, one in each apparatus. During a session, food pellets are thrown into the apparatus at a rate of about $3 / \mathrm{min}$. At first, the animals stay close to the wall. With time, however, most rats will orient towards a food pellet when it falls and then go to it, even if it lands in the center of the apparatus. Since a pellet is available about every $20 \mathrm{sec}$, the rat spends most of its time moving. The criteria for completion of training are that the rat must chase food pellets in all 4 apparatuses, and must spend at least $75 \%$ of the time moving. Training takes as long as 3 weeks, mainly because the rats are reluctant to move away from the walls of the large apparatuses. It is important to note that the rats are thoroughly familiar with all 4 apparatuses before any recordings are made. The purpose of overtraining is to minimize the likelihood that the first cell or cells recorded from a given animal will systematically differ from other cells because the animal is in an active state of learning.

\section{Electrode preparation and surgery}

We use a drivable 10 wire $(25.4 \mu \mathrm{m}$ diam each) electrode array whose construction is described elsewhere (Kubie, 1984). In preparation for surgery, rats are anesthetized with pentobarbital $(40 \mathrm{mg} / \mathrm{kg})$. Before implantation, the electrode array is sterilized with betadinc and the exposed wires embedded in a small drop of Carbowax. The Carbowax allows the wires to be advanced as a unit during implantation. After a few minutes in the brain, the wax dissolves and the individual wires separate a little. The rat is placed in a Kopf stereotaxic frame, the skull is exposed, and securing screws are placed over the right olfactory bulb, the left frontal cortex, and the left cerebellar hemisphere. A $2 \mathrm{~mm}$ hole is made in the lateral region of the right parietal bone to expose the dura. (All implantations are made in the right side.) The electrode bundle is aligned normal to the skull; this requires a $10^{\circ}$ angle in the coronal plane, with the tip pointing medially. The electrode is aimed at the hilus of the dentate gyrus $(3.0 \mathrm{~mm}$ anterior and $1.7 \mathrm{~mm}$ dorsal to ear-bar 0 , and $2.8 \mathrm{~mm}$ lateral to the midline) according to the atlas of Pellegrino et al. (1967). The bundle tip is driven $1.7 \mathrm{~mm}$ below the dura. At this depth, the tip of the longest wire is above the CAl pyramidal cell layer. Sterile petroleum jelly is applied to the exposed surface of the brain and the guide tubing to seal the skull opening. Dental acrylic (Turotech, Wynnewood, PA) is applied over the jelly and around the guide tubing. Finally, the rest of the exposed skull is covered with Grip cement (Ranson and Randolph Ceramics, Maumee, $\mathrm{OH}$ ), and the bases of the 3 drive screws of the electrode are cemented to the skull. The rat is given $3-5 \mathrm{~d}$ to recover after surgery.

\section{Data acquisition}

Recording environment. A controlled cue environment is created with a circular curtain that is $213 \mathrm{~cm}$ long and $213 \mathrm{~cm}$ in diameter. The bottom of the curtain is just above the floor; the top is $70 \mathrm{~cm}$ below the laboratory cciling. Several other items are hung from beams mounted $240 \mathrm{~cm}$ above the floor. The TV camera used to track the animal's position is centered in the curtain ring. Slightly to one side is a multichannel mercury commutator. The commutator and the attached cable supply power to FET source followers, lead the outputs of the FETs 
to the amplifier, and also supply power to 2 small lights mounted on the electrode carrier. Finally, there are four $15 \mathrm{~W}$ incandescent bulbs in $22 \mathrm{~cm}$ hemispherical reflectors mounted at the corners of a $90 \mathrm{~cm}$ square around the TV camera. These lamps are the main source of illumination.

Electrophysiological identification of cells. Ammon's horn neurons in freely moving rats are classified as complex-spike or theta cells (Ranck, 1973). Complex-spike cells may be recognized in several ways. First, they sometimes generate the bursts of action potentials with gradually decreasing amplitude for which they are named. Complex-spike cells also produce single action potentials the same size as the first action potential in a complex burst; single action potentials, in fact, arc much more common. Second, they tend to be silent under some circumstances. Third, the duration of the negative component of extracellular spikes (simple or complex) is characteristically greater than about $0.3 \mathrm{msec}$ (Fox and Ranck, 1981; Berger et al., 1983).

Theta cells are units that approximately double their firing rate whenever the hippocampal EEG is in the theta state (5-9 Hz sine-like waves) while the animal is moving. In contrast to complex-spike cells, theta cells never show complex bursts, are rarely silent, and have a shorterduration negative phase $(<0.25 \mathrm{msec})$. The firing of both complex-spike and theta cells is correlated with the phase of the hippocampal theta rhythm (Fox et al., 1986).

Complex-spike cells appear to be pyramidal cells, whereas theta cells are likely local interneurons (Fox and Ranck, 1975, 1981; Berger et al., 1983). Thus, electrophysiological criteria allow us to tentatively identify cells according to their anatomical class. To histologically confirm that recorded cells were found at appropriate depths, the longest electrode is used to produce a small DC lesion at the bottom of the single pass through the hippocampus. Forty $\mu \mathrm{m}$ frozen sections stained with cresyl violet are examined to find the course and bottom of the electrode track. The distance from the bottom of the track to the cell layers of the hippocampus is compared to the estimated depth at which each cell was found. In general, there is excellent agreement between the measured location of the pyramidal cell layers and the position of complex-spike cells calculated from advances of the electrode array.

Recording equipment. To minimize recording artifacts, the cable from the electrode array to the commutator contains FET source followers at the head end. The low-output impedance signals from the FETs are fed through the commutator, amplified, and sent to 2 time/amplitude discriminators (Model DIS-1; Bak Electronics, Rockville, MD) arranged in series. The first discriminator is set to accept the peak negativity of the chosen unit. The amplitude window is wide enough to accept the first and 1 or 2 of the next negative peaks of a complex-spike burst; later peaks are likely to be rejected. Acceptance pulses from the first discriminator start the internal delay of the second, whose window is set for the positive phase of the extracellular spike. A spike is counted when the second discriminator produces an acceptance pulse. Two windows yield excellent selectivity among units, and also serve to reject almost all artifacts.

Cell sample. Before a set of recording sessions is started, each of the 10 electrodes is checked for units. If no well-isolated units are found, the array is advanced by 30 or $60 \mu \mathrm{m}$. The animal is then returned to its home cage for at least $4 \mathrm{hr}$ to ensure that movement of the brain relative to the electrodes is complete before another check is made.

If a well-isolated unit is seen, its type is determined. If it is not a complex-spike cell, it is often rejected. We are mainly interested in complex-spike cells because place cells are a subset of complex-spike cells (O'Keefe, 1979). If the unit is a complex-spike cell, it is screened by placing the animal successively into the small cylinder and small rectangle, and it is used only if direct observation reveals a firing field in either or both apparatuses; the firing rate in putative fields must be greater than about 1 action potential (AP)/sec. This screening helps to detect potentially interfering units that are quiet in 1 of the 2 small chambers. To enhance the likelihood that the recording will be stable for the several hours needed to complete an experiment, the rat is returned to its home cage for half an hour. If, after the delay, the waveform is unchanged and is still accepted with the same discriminator settings, the unit is used. If this test fails, the delay/check cycle is repeated.

Most of the data presented here come from 40 complex-spike cells that were recorded under fixed conditions in the small cylinder. The sample was obtained from 17 rats; the number of cells per rat ranged from 1 to 6 . In general, many sessions were done for each cell in the sample. On the average, each unit was recorded for a total of $66 \mathrm{~min}$,
44 of which were in the small cylinder. Examples of rate maps were chosen from the complete set of sessions. By contrast, population parameters (e.g., average field size) were estimated from one 16 min session for each of the 40 cells. To eliminate selection bias, each of these sessions was the first done on a given cell in the small cylinder. Of the 40 complexspike cells, 14 were recorded from the CA1 pyramidal layer; the remainder were from $\mathrm{CA} 3 / 4$.

Data-acquisition system. Figure 1 is a block diagram of our dataacquisition system, which may conveniently be divided into 3 subsystems:

1. Electrophysiological recording set-up: (a) the electrode array, cable, commutator, and amplifier; (b) the delay line and the 2 window discriminators; (c) a 4-bit counter to accumulate the number of spikes accepted by the discriminators during each sample interval (1/60th sec). Since the counter will not saturate until 15 spikes have been fired in a sample interval, the unit could fire at $900 \mathrm{AP} / \mathrm{sec}$ with no missed spikes. This rate is well in excess of the highest rates seen (about $200 \mathrm{AP} / \mathrm{sec}$ for very brief intervals). The $60 \mathrm{~Hz}$ rate is dictated by the video field rate.

2. Location detector: (a) two miniature ("grain of wheat") incandescent bulbs (Microlamps Inc., Lafox, IL) are mounted $1 \mathrm{~cm}$ above the rat's head on the electrode carrier. The bulbs are set behind the carrier and are about $0.5 \mathrm{~cm}$ apart; each is medial to an ear. The bulbs are DC powered through the commutator and cable; (b) a television camera mounted $240 \mathrm{~cm}$ above the floor and centered in the curtains. With the lens used, the area viewed by the camera is $223 \times 180 \mathrm{~cm}$; (c) a threshold device that detects the time, in each TV field, that the video scan moves across the headlights, which are the brightest objects in the field; (d) means to convert the time of headlight detection into a pair of 8-bit binary numbers that represent the $X$ and $Y$ coordinates of the headlights. Only the most significant 6 bits of each coordinate are kept, so that the headlights are located in a $64 \times 64$ grid of picture elements (pixels). The size of each pixel is $3.5(X) \times 2.8(Y) \mathrm{cm}$; (e) if the headlights are not detected during a TV field, the $Y$ coordinate for the sample is set to zero. Zero is an impossible value for a good sample, since it is outside the apparatus.

3. Microcomputer plus interface. Values for the 6-bit $X$ and $Y$ location registers and the 4-bit spike counter (16 bits in all) are transferred to the computer at the end of each video field. The interface is operated in 1 of 2 ways: (a) in calibration mode, numbers representing the location of a test light are displayed on the computer screen once per second. This allows the aim of the TV camera to be checked, and also permits the recording chamber to be reproducibly placed relative to the TV field; (b) in data-acquisition mode, the computer is interrupted each $1 / 60$ th of a second, at the time of the vertical synchronization pulse between fields. Samples are stored sequentially in memory and are sent as a time series to a floppy disk at the end of the experimental session. Data analysis is done later ("off line"). A session lasts 8 or, more often, $16 \mathrm{~min}$; a 16 min session consists of 57,600 samples.

Limitations of the data-acquisition method. This method for measuring spatial spike artivity has the advantage that computation is unnecessary in order to decide which spikes are associated with each sample of the animal's position, since position and spike count are stored for each video field as a 16-bit number. On the other hand, the time when a spike occurs cannot be specified with better than $16.7 \mathrm{msec}$ resolution. For most single cell work, this temporal resolution would be inadequate. In the present case, however, we are interested mainly in the time-averaged spatial distribution of firing rate, and it would not help to measure the time of spikes more precisely than we can know where the animal was at a given moment.

There are 3 sources of error that the tracking system introduces into measurements of the animal's position, even assuming the animal is not moving. The first comes from the use of 2 headlights. If spatial resolution were the only problem, a single bulb would be better, but 2 lights reduce the number of samples in which no light detection occurs. Since the 2 bulbs are $0.5 \mathrm{~cm}$ apart, this error is on the order of 0.2 pixels.

The second and third errors involve, respectively, jitter in the $X$ and $Y$ positions of a fixed light near the boundaries of each pixel. The $Y$ jitter, which is on the order of $1 / 4$ pixel, comes from the interlaced scan pattern of the TV camera. In alternate fields ( 2 of which make up a frame), the position of each line is displaced by the width of a line. This error is only $1 / 4$ pixel because the lowest 2 bits from the 8-bit $Y$ counter are discarded. It can be eliminated by sampling every other field (which would halve the temporal resolution) or by using a camera that does 
Figure 1. Block diagram of the dataacquisition system. The box marked Detect end of frame derives $60 \mathrm{~Hz}$ timing pulses from the video signal. The pulse is used to trigger (interrupt) the computer to read the data from the last vidco scan. After a delay, the same pulse is used to reset the spike and position registers, so that they are available to hold information from the upcoming scan. The $X$ and $Y$ headlight coordinates are obtained by converting the time of headlight detection during the TV field to 6-bit numbers. This information, along with a count of the number of spikes ( 4 bits) fired during the TV field make up a single sample. Samples are stored in the computer as a time series, and put onto a disk at the end of the session for data analysis. Additional description in text.

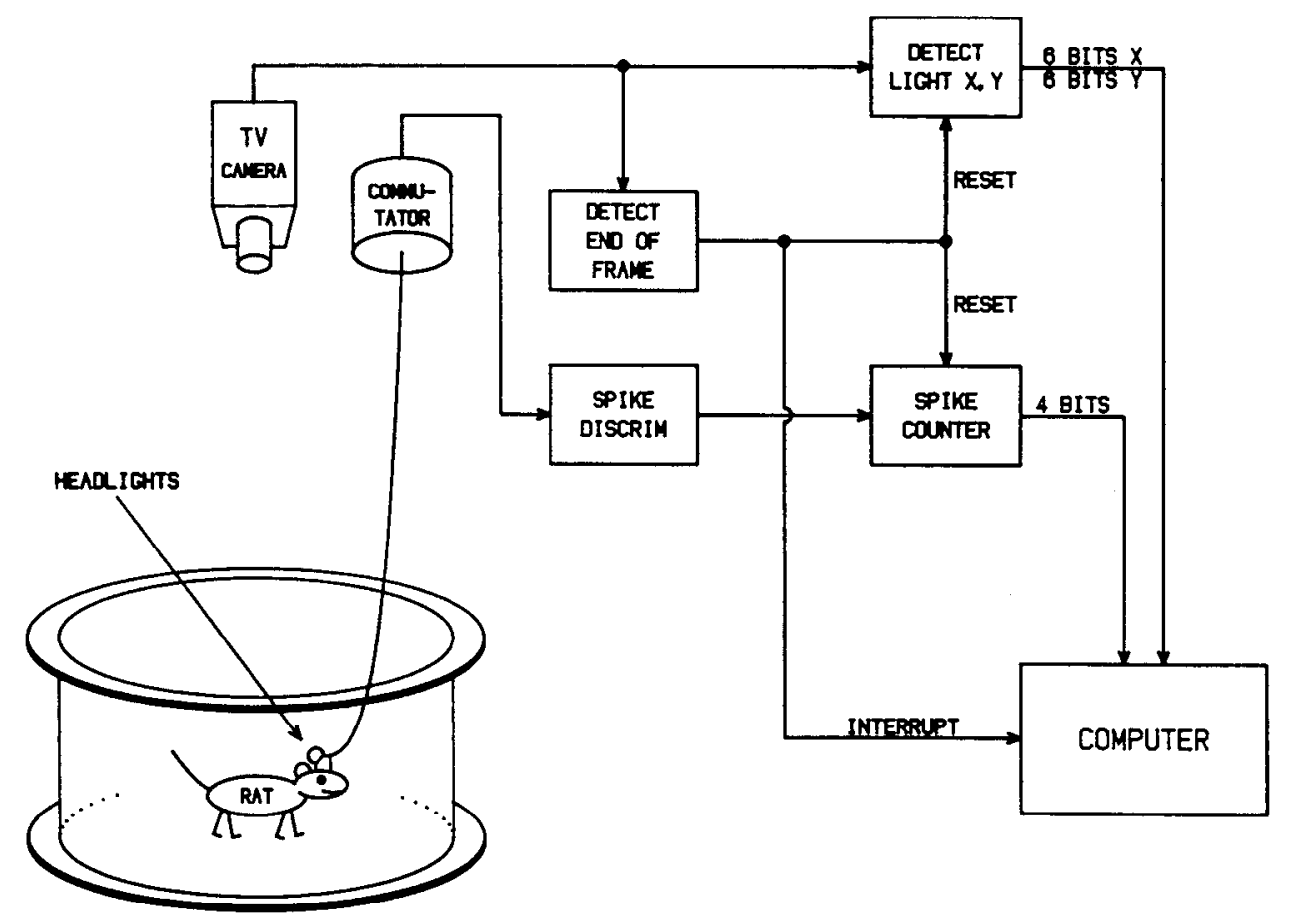

not interlace. The $X$ jitter occurs because the $5 \mathrm{MHz}$ clock is not synchronized to the start of each line. As a result, the apparent time of headlight detection can vary by $1 / 2$ clock cycle. Since the 2 least significant bits of the 8-bit $X$ counter are ignored, this error is also $1 / 4$ pixel.

Running a recording session. Before each session, a new piece of paper is put on the laboratory floor to eliminate constant cues from the floor. The selected apparatus is put on the paper and positioned according to the coordinates of a test light set on a known spot of the apparatus. Next, the animal is brought inside the curtains and the recording/power cable is attached to the selected electrode. The unit's waveform is checked against established discriminator settings. The headlights are turned on, the threshold of the headlight detector is adjusted, and the session is started. During the session, one of us watches the rat on a TV monitor, listens to the unit activity, and throws about 3 food pellets per minute over the curtains. If too much electrical noise is seen or if the cable comes loose, the session is terminated and the data discarded.

\section{Analysis and presentation of data}

Unpacking of serial samples. The first step in analysis is to transform serial samples into spikes-in-location and time-in-location distributions. The first byte of each sample contains the 2 most significant spike count bits and the 6 bits of $Y$ position data. The second byte contains the 2 least significant spike count bits and the 6 bits of $X$ position data. The $X$ and $Y$ positions are used as indices into a $64 \times 64$ time-inlocation array and a $64 \times 64$ spikes-in-location array. The indexed element in the time array is incremented by 1 ; the same element in the spike array is incremented by the number of spikes fired during the $1 / 60$ th sec sample interval. This sequence is repeated for all the samples in the time series. A $64 \times 64$ rate array is then filled by dividing the time array into the spike array on an element-by-element basis. Unvisited pixels are marked by setting the appropriate elements of the rate array to -1 .

Recognizable errors in the data are found and eliminated before the firing rate array is constructed. A zero $Y$ coordinate means that the headlights were not detected during the sample. With no detection, the animal's position is indeterminate, and spikes fired during the sample are ignored. Nondetects usually make up a few $(<5)$ percent of the total sample and are generally due to occlusion of the headlights by the recording cable, although unusual postures on the part of the animal can also produce them.
The second class of recognizable errors involves samples for which the rat was apparently outside of the apparatus. Samples outside the apparatus are found by comparing each sample to a template of the apparatus. The number of "displacement" errors is the difference between the total number of samples and the sum of the "good" and nondetect samples. Most displacement errors are caused by setting the threshold of the headlight detector too close to background light levels; if a relatively bright region is found by the threshold device before the headlights are detected, an "impossible" position can be registered. This sort of error implies there may be samples inside the apparatus that are displaced from their proper location. With experience, we have reduced the occurrence of displacement errors to near zero.

Presentation of data. Most of the figures in this paper are color-coded maps that summarize a time, a spike, or a rate array from a single experimental session. For each map type, increasing values of the relevant variable are coded in the following order: yellow, orange, red, green, blue, and purple. We use this split spectrum because it produces spike and rate maps in which firing fields show up as dark areas on a yellow background. The correspondence between the numerical value of an array element and the color of the displayed pixel is established on a relative rather than absolute basis. The method calculates a target number of pixels to be printed in each color, and then finds the appropriate breakpoints between color categories.

The time-in-location array is transformed into a map in which the number of pixels printed in each of the 6 colors is as close to the same as possible. Regions of space that were never visited are left white. Since the rats enter almost every accessible pixel during a session, the area of the apparatus appears as a colored region.

The methods for producing spike and rate maps are much the same, except that yellow is used for visited pixels in which no action potentials were fired, so that finite spike counts or firing rates are coded with 5 instead of 6 colors. Using yellow to code "zero" pixels is valuable because the frequent occurrence of such pixels makes firing fields stand out clearly; for instance, in the firing rate map of Figure $2 C$, the field is at 7 o'clock. For spike and rate maps, the numbers of pixels coded as other than yellow are made unequal; the number of pixels of a given color is set to 0.8 times the number of pixels in the next lower color. This means (with 5 colors) that the number of orange (lowest spike count or rate) pixels is about twice that of purple (highest spike count or rate) pixels. This enhances the contrast of the most intense part of a firing field against the rest of the field. 


\section{Results}

Location-specific firing of a "typical" complex-spike cell The outcome of a 16 min recording session is shown in Figure 2 and Table 1 . Figure $2, A-C$, shows, respectively, color-coded maps of time-in-location, spikes-in-location, and firing rate arrays from a $16 \mathrm{~min}$ session in the small cylinder, arranged as a division to show how the rate map is derived from the primary data. Information on the numerical values represented by the colors in Figure 2 is given in Table 1.

The firing field for the cell recorded in this session appears as a circumscribed, continuous dark region at 7 o'clock in the rate map of Figure $2 C$. In this case and almost all others, the covariation of position and firing is strong enough that the location, shape, and approximate size of firing fields are evident from the inspection of rate maps. The specificity of spatial firing is sufficiently great for the field to still be evident in a 2-valued firing rate map in which the only distinction would be between pixels with zero and those with greater-than-zero firing rates.

Within the field itself, the firing rate falls off steeply in all directions from the apparent field center. Table $1 \mathrm{C}$ shows that the median firing rate for each color category is about 2 times that of the next lower category; the median rate in the fastest (purple) category is 23.5 times as great as that for the slowest nonzero (red) category. The decline from the peak rate of about 30 to $0 \mathrm{AP} / \mathrm{sec}$ takes place over a distance of approximately 20 $\mathrm{cm}$. Thus, the field is a rather sharp peak rather than a broad mesa of firing. In addition, with only 6 firing rate categories, the rate contours of the field appear to be fairly smooth. The properties of steep firing rate gradients and smooth firing rate contours are shared by most place cells.

The development of the field structure with recording time is illustrated in Figure 3 . Figure $3 A$ is a rate map for the first 4 min of the session shown in Figure 2. The broad outlines of the firing field are apparent, but the rate contours are very noisy. Note also that the rat did not visit all of the accessible pixels in the $4 \mathrm{~min}$, as shown by the white patches and the somewhat irregular outline of the cylinder. Figure $3 B$ is a rate map for the middle $8 \mathrm{~min}(5-12)$ of the same session. After $8 \mathrm{~min}$, the

\begin{tabular}{lcccc}
\hline $\begin{array}{l}\text { Table 1. } \\
\text { Figure 3 }\end{array}$ & Numerical values for the color categories in the maps of \\
$\begin{array}{l}\text { Color } \\
\text { category }\end{array}$ & $\begin{array}{l}\text { No. of } \\
\text { pixels }\end{array}$ & $\begin{array}{l}\text { Minimum } \\
\text { pixel }\end{array}$ & $\begin{array}{l}\text { Median } \\
\text { pixel }\end{array}$ & $\begin{array}{l}\text { Maximum } \\
\text { pixel }\end{array}$ \\
\hline $\begin{array}{l}\text { A. Time-in-location (sec) } \\
\text { Yellow }\end{array}$ & 87 & & & \\
Orange & 84 & 0.017 & 0.417 & 0.733 \\
Red & 86 & 1.133 & 1.267 & 1.417 \\
Green & 86 & 1.433 & 1.683 & 1.967 \\
Blue & 85 & 1.983 & 2.250 & 2.683 \\
Purple & 85 & 2.700 & 3.433 & 24.050 \\
B. Number of spikes & & & \\
Yellow & 334 & 0 & 0 & 0 \\
Orange & 53 & 1 & 1 & 2 \\
Red & 44 & 3 & 4 & 7 \\
Green & 33 & 8 & 11 & 15 \\
Blue & 26 & 16 & 19 & 26 \\
Purple & 23 & 27 & 34 & 75 \\
C. Firing rate (AP/sec) & & & \\
Yellow & 334 & 0.00 & 0.00 & 0.00 \\
Orange & 53 & 0.19 & 0.77 & 1.58 \\
Red & 43 & 1.60 & 2.79 & 3.81 \\
Green & 34 & 3.82 & 6.12 & 8.57 \\
Blue & 27 & 8.64 & 10.74 & 13.99 \\
Purple & 22 & 14.21 & 18.10 & 29.23 \\
\hline
\end{tabular}

sampling of the apparatus area is nearly complete. In parallel, the firing rate contours have become considerably smoother. With a total of $16 \mathrm{~min}$ of recording (Fig. $3 C$; the map is from a second session done with the same cell), all parts of the apparatus have been visited and the firing rate contours have once again become simpler. Combining data from the two $16 \mathrm{~min}$ scssions summarized in Figures $2 C$ and $3 C$ produced the firing rate map of Figure $3 D$. After 32 min of sampling, the field is composed of a series of concentric bands; the rate decreases monotonically in all directions away from the center.

\footnotetext{
Figure 2. The 3 maps obtained from session R11S2B16 (Unit R11U1; CA3/4). Session names give the rat's number, the number of the session (started at 1 for each rat), and the duration of the session in minutes. $A$, Time-in-location map. $B$, Spike map. The firing field at 7 o'clock is evident, even without normalization by the time spent in each pixel. $C$, Firing rate map. After dividing the spike array by the firing rate array on a pixelby-pixel basis, concentric color rings that denote a single peak of intense activity are apparent. Numerical values represented by the colors in the maps are given in Table 1.
}

Figure 3. Firing rate maps for total recording times of 4, 8, 16, and $32 \mathrm{~min}$. The cell is the same one whose spatial firing pattern was shown in Figure 2. As the total recording time increases, more and more of the apparatus area is visited by the rat. In parallel, the apparent size of the firing field at 7 o'clock grows and the firing rate contours within the field become progressively simpler. $A$, Firing rate map for the first 4 min of session R11S2B16. Median firing rates for colors: yellow, 0.0; orange, 2.86; red, 7.50; green, 10.9; blue, 16.4; purple, 30.0 AP/sec. For the rest of the firing rate maps in this paper, median firing rates for color categories are listed in ascending order without repeating the color sequence. $B$, Firing rate map for minutes $5-12$ of session R1 1S2B16. Median firing rates: $0.0 ; 1.43 ; 4.15 ; 8.57 ; 15.5 ; 25.2$. $C$, Firing rate map for a different 16 min recording session (R11S1B16) done on the same cell as R11S2B16. Median firing rates: $0.0 ; 0.54 ; 1.49 ; 3.82 ; 7.50 ; 15.0$. $D$, Firing rate map that results when the data from the two $16 \mathrm{~min}$ sessions were combined to produce a single sequence, which was then treated as a 32 min session. Median firing rates: $0.0 ; 0.32 ; 1.13 ; 2.79 ; 7.43 ; 13.5$.

Figure 4. The spatial firing patterns of place cells are stable for days. Rate maps in $A$ and $B$ were obtained from 2 cells recordable on the same wire. The session in $A$ was done $1 \mathrm{hr}$ before that in $B$; the 2 spike trains were separated with the window discriminators. Rate maps in $C$ and $D$ were obtained 6 d later from the same 2 cells. $A$, Session R8S25B8; unit R8U5A (CA1). Median rates: $0.0 ; 0.49 ; 1.40 ; 4.62 ; 10.9 ; 21.6 . B$, Session R8S26B8; unit R8U5B (CA1). Median rates: $0.0 ; 0.74 ; 1.80 ; 3.13 ; 6.00 ; 15.0$. C, Session R8S29B8; unit R8U5A. Median rates: 0.0; 0.48; 1.33 ; $2.22 ; 4.80 ; 9.73$. $D$, Session R8S30B8; unit R8U5B. Median rates: $0.0 ; 0.71 ; 1.71 ; 2.76 ; 4.69 ; 7.20$.

Figure 5. Examples of the classes of spatial firing patterns seen in the small cylinder. $A$, A roughly circular field similar, except in position, to the field in Figure $2 C . B$, Large, elliptical field in the center of the apparatus. $C$, Elliptical field that touches the apparatus wall. $D$, Crescentic field. $E$, Rate map for a cell that was virtually silent in the small cylinder. The same cell had a strong firing field in the small rectangle. $F$, Rate map with 2 clear-cut firing fields. The question of whether such firing patterns are generated by 1 or 2 cells is considered in the text. 
B

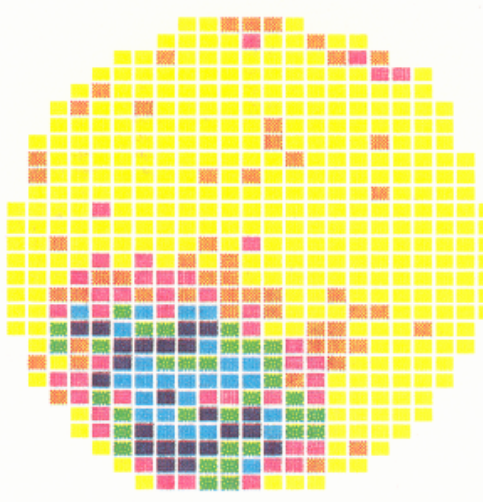

A

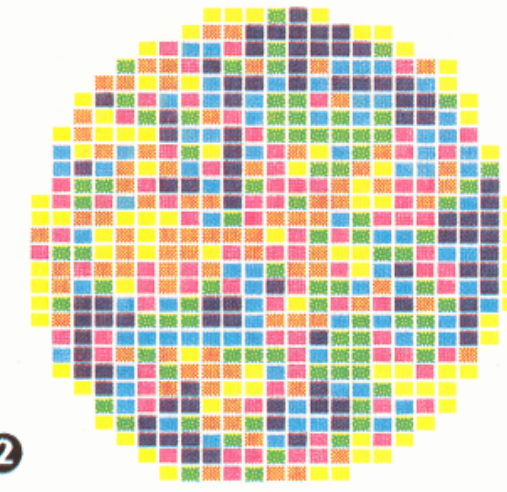

A
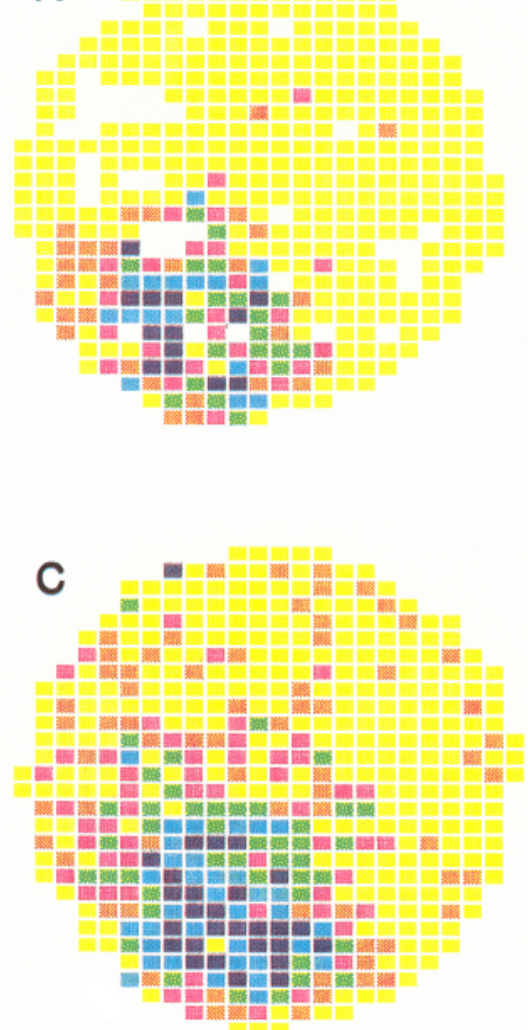
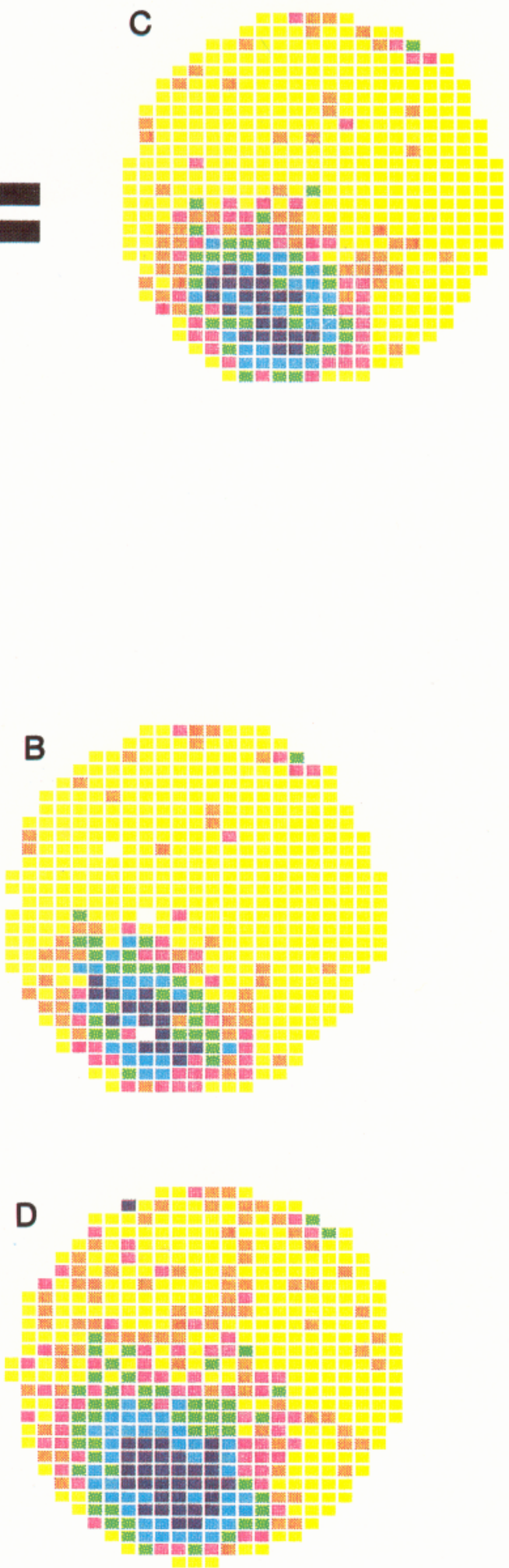

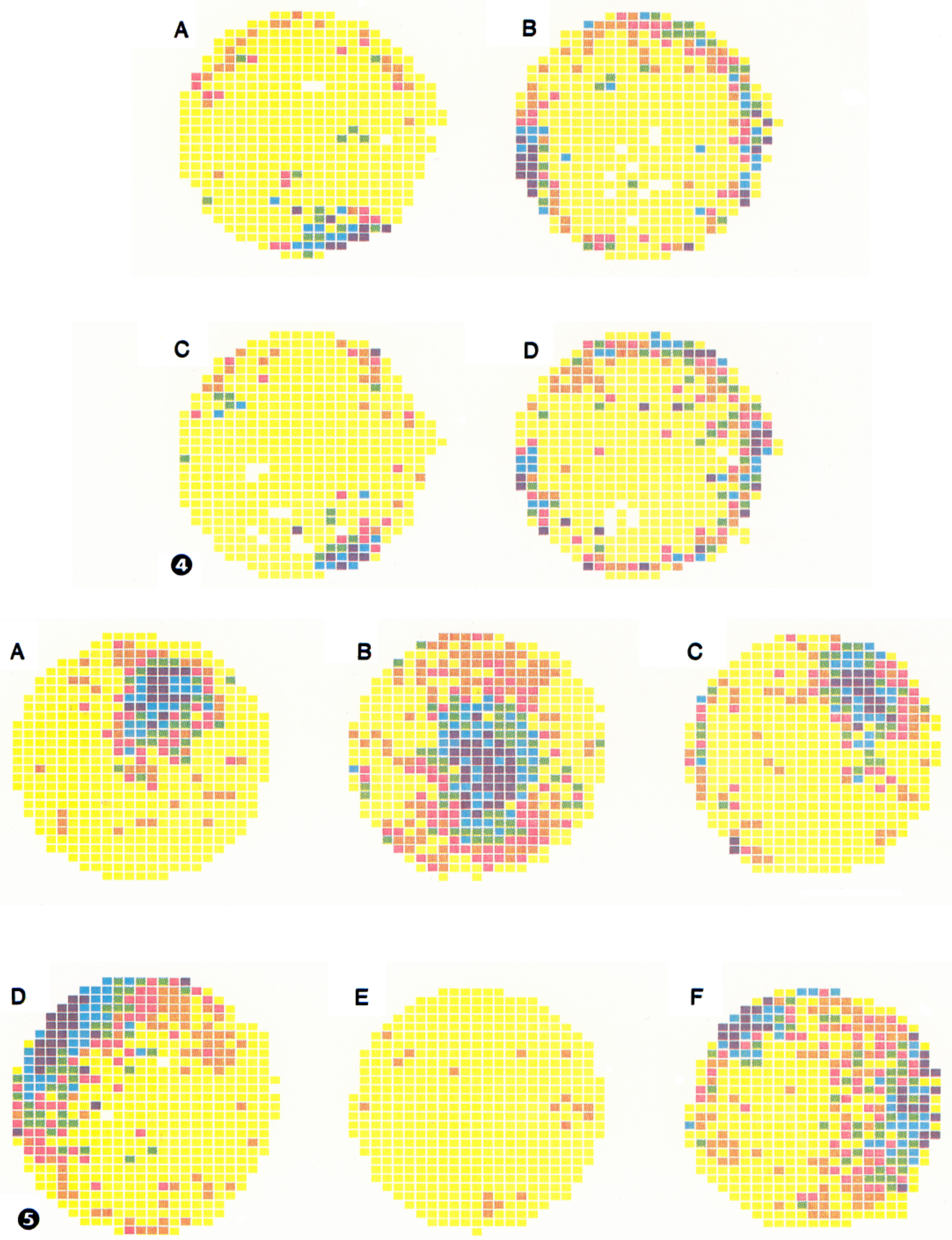
Table 2. Firing rates for 6 different cells recorded in the small cylinder

\begin{tabular}{llllllllll} 
& & & & \multicolumn{7}{c}{ Mcdian firing ratc (AP/scc) } \\
\cline { 6 - 11 } Map & Session & Cell & Position & Y & O & R & G & B & P \\
\hline A & R3S20B16 & R3U4 & CAl & 0.0 & 0.45 & 1.73 & 3.90 & 7.36 & 18.5 \\
B & R4S4B16 & R4U3 & CA3/4 & 0.0 & 0.52 & 1.45 & 3.53 & 10.9 & 19.1 \\
C & R3S48B16 & R3U8 & CA3/4 & 0.0 & 0.64 & 1.62 & 2.86 & 6.92 & 13.5 \\
D & R58S3B16 & R58U1 & CA1 & 0.0 & 0.66 & 2.00 & 4.77 & 8.83 & 16.8 \\
E & R2S8B16B & R2U2B & CA3/4 & 0.0 & 0.27 & - & - & - & $-^{a}$ \\
F & R3S35B16 & R3U6 & CA3/4 & 0.0 & 0.55 & 1.26 & 2.57 & 4.88 & $11.4^{b}$
\end{tabular}

${ }^{a}$ Because of the low overall firing rate in this session, all of the pixels for which the firing rate was greater than zero are plotted in orange.

${ }^{b}$ Firing rates are for both fields. The average rate in the field at 3 o'clock was $1.90 \mathrm{AP} / \mathrm{sec}$. The average rate in the field at 1 o'clock was $4.59 \mathrm{AP} / \mathrm{sec}$. The difference in the rates is mainly a function of the large number of low firing rate pixels in the field at 3 o'clock; the peak (field center) rates are similar $(10.4 \mathrm{vs} 13.0 \mathrm{AP} / \mathrm{sec})$.

The progressive smoothing of firing rate contours with increased sampling time is thus not complctc after $16 \mathrm{~min}$ of recording. If the only consideration were to accurately capture the spatial firing patterns of place cells, it is clear that it would be better to record for 32 min or longer. It is equally clear, however, that $16 \mathrm{~min}$ of recording is sufficient to get a good idea of the location, shape, and size of the firing field, and that $16 \mathrm{~min}$ is therefore a reasonable compromise, given the need to record from the same cell under varying conditions (see Muller and Kubie, 1987). A comparison of the spike (Fig. 2B) and rate (Fig. $2 C$ ) maps for the sample session also suggests that 16 min is adequate to get a good picture of the firing rate distribution. The exact correspondence of yellow pixels in the 2 maps is a result of using yellow to code zero spikes and zero firing rate. Note, however, that the contours in the rate map are smoother. It follows that sufficient time was spent in each pixel for a fairly accurate estimate of firing rate to have been obtained. This is despite the fact that the animal spent relatively little time in the region of the field center, as can be seen from the time-in-location map of Figure $2 A$.

\section{Temporal stability of firing fields}

The rate maps in Figures $2 C$ and $3 C$ demonstrate that firing fields are stationary from session to session. The pixel-by-pixel coefficient of correlation between the 2 firing patterns was 0.70 , so that about half the variance of firing between the 2 sessions was accounted for by place alone. The strength of this effect is emphasized when we remember that the calculated correlation treats each pixel as an independent sample, and that the orderliness of the field is ignored. It should also be remembered that the animal was removed from the apparatus between the 2 sessions, during which time the floor paper of the cylinder was changed. This suggests that the constancy of firing need not depend on cues local to the region of the field, although it is possible that such cues are important during a single recording session.

Figure 4 demonstrates longer-term temporal stability; it shows fields from 2 cells that were recordable from a single wire at the same time. The 2 spike trains were separated with window discriminators and with alternating sessions for each cell. The maps of Figurc $4, A, B$, show the firing pattern of the 2 cells in 2 sessions done $1 \mathrm{hr}$ apart. The very similar maps in Figure 4, $C, D$, are for the same 2 cells $6 \mathrm{~d}$ later; the correlation coefficients between $4, A$ and $C$, and $4, B$ and $D$, were, respectively, 0.70 and 0.45 . This experiment has been repeated by us many times, usually with intervals of 1 or $2 \mathrm{~d}$, with similar results. At the cxtrcmc, Bcst and Thompson (1984) found a single cell to have the same firing field for $153 \mathrm{~d}$.

\section{Dwell time and place cell firing}

The tendency of rats to spend less time in open areas away from the wall is visible in the time-in-location map of Figure $2 A$, although the effect is not very pronounced in this case. Some rats also preferred a rather broad $\left(>90^{\circ}\right)$ wedge of the apparatus, which varied from animal to animal. It is therefore important to check to see whether place cells tend to fire in the preferred regions; a positive answer would indicate an important dependency of place cell firing on a behavioral variable other than position. To this end, the correlation coefficient between the time-in-location and firing rate arrays was calculated for each cell that was considered to have a firing field (the definition of a field is given below). The mean coefficient was zero within experimental error $(r=0.026$; range, -0.25 to +0.26$)$. A few cells showed significant positive or negative correlations, but the bulk of the correlations were near zero; a chi-squared test did not reject the hypothesis that the correlation coefficients were normally distributed around the mean. Thus, the average results indicate that spatial firing is independent of dwell time or preference for a certain region.

\section{Shapes of firing fields}

In Figure 5, we show rate maps for 6 different cells recorded in the small cylinder. Firing rates for the cells are given in Table 2. These maps are intended as examples of the various firing rate patterns seen. The field in Figure $5 A$ is similar, except in position, to the one considered in detail above. To a first approximation, it is radially symmetric, with reasonably clear concentric iso-firing rate contours. The field in Figure $5 B$ appears elliptical, and is one of the largest seen. Figure $5 C$ shows a field that is approximated by an ellipse whose major axis is parallel to the line connecting 3 and 12 o'clock. Thus, fields can be elliptical or circular, even if they encroach on the wall.

The field illustrated in Figure $5 D$ stands in marked contrast to the others discussed so far. It is best described as crescentlike, and not as a convex field truncated by the wall. As for most cells with fields of this shape, the most active part of the field is right up against the apparatus wall. There are two 3-dimensional firing patterns that are consistent with the observed 2-dimensional pattern. On the one hand, it is possible that the cell in Figure $5 D$ is firing in association with the crescent-shaped 
part of the apparatus floor, so that the firing pattern is, after all, best considered to be 2-dimensional. The implication is that the spatial firing distribution reflects the local structure of the environment, as well as the position within the environment. On the other hand, it is possible that the crescent-shaped firing region is the projection of a firing field that extends upwards along the cylinder wall. To distinguish between these hypotheses will require that the animal's head position be measured in 3 dimensions. Nevertheless, the existence of edge-conforming fields means that the apparatus boundary is treated specially. In Figure 6 we give examples of edge-conforming fields recorded in other apparatuses to show that these interesting patterns are not peculiar to the small cylinder. Note that the edge fields in the rectangles are linear.

The rate map in Figure $5 E$ shows another characteristic pattern of spatial firing-or more accurately, its absence. The cell produced a total of $14 \mathrm{AP}$ in $16 \mathrm{~min}$, for an average rate of $0.015 \mathrm{AP} / \mathrm{sec}$. It was recognized as a place unit only because it had a clear field in the small rectangle. The existence of "silent" cells in a given apparatus suggests that different subsets of the place cell population may be used to represent different environments, an issue that will be dealt with more fully in the following paper (Muller and Kubie, 1987). The only complexspike cells that were seen to have spatially homogeneous firing distributions were those for which the firing rate everywhere approached zero.

The map in Figure $5 F$ gives the final, prototypical firing pattern. The new feature in this map is the presence of 2 distinct regions of intense activity. Such a pattern would be generated by an individual neuron with 2 fields, or by 2 neurons with very similar spike waveforms at the recording electrode. The question of whether a single place cell may have more than 1 field should be investigated using the stereotrode technique of McNaughton et al. (1983b). Since we used single electrodes, we cannot be sure that any of the maps with 2 fields were obtained from single units. Nevertheless, it is our strong impression that a single place cell may have 2 (or more) firing fields. Figures 7 and 8 show our best evidence that this is true. The experiment illustrated in Figure 7 demonstrates the ability to discriminate between 2 rather similar spike waveforms. Figure $7 A$ is a multiple-sweep picture showing action potentials recorded from both cells. When the discriminators were opened to accept both spikes, the map of Figure $7 B$ was seen. In separate sessions, the discriminators were set to accept only the larger (Fig. 7C) or smaller (Fig. 7D) action potentials, showing that the pattern obtained without discrimination was a composite of the spatial firing of 2 independent cells. The scattered firing at 10 o'clock in Figure $7 C^{\prime}$ and at 7:30 o'clock in Figure $7 D$ is "spillover" due to imperfect discrimination. Figurc 7 demonstrates good control over which action potentials are accepted. It also shows that neighboring cells can have widely separated fields in the same environment.

The experiment illustrated in Figure 8 tested the notion that an individual cell can have 2 fields. Figure $8 A$ is a map with 2 fields recorded in the large cylinder. Figure $8, B 1$ and $B 2$, shows multiple-sweep traces obtained from the target neuron when the animal was in the 12 and 7 o'clock fields, respectively. The electrode array was then advanced by about $15 \mu \mathrm{m}$ and the traces in Figure 8,C1,C2, were taken. The 2 waveforms are the same as in Figure $8, B 1, B 2$, but reduced in amplitude by about $30 \%$. A second $15 \mu \mathrm{m}$ advance led to another $30 \%$ decrease of the spike amplitude in both fields (Fig. 8, D1, D2), again with no change in waveform. This result suggests, but does not prove, that a single unit was involved. Given the parallel arrangement of pyramidal (place) cells, the data in Figure 8 are also compatible with the idea that the electrode ran parallel to, and halfway between, 2 pyramidal cells. The main argument in favor of the single cell explanation is its simplicity; there is no need to imagine very particular relationships between the electrode and the cell matrix.

In summary, it is possible to divide spatial firing patterns in the small cylinder into several classes. The occurrence of each type, as judged by eye from the appearance of the field (the largest if there was more than one) was as follows: 11 crescentlike; 18 circular; 4 elliptical; 6 no field; and 1 uncategorized. Of the 40 cells, 6 had no field, 25 had 1 field, and 9 had 2 fields.

\section{Quantitative measures of place cell firing}

Pictorial representations such as rate maps are useful for illustrating the overall pattern of spatial activity. Maps are also valuable for detecting invariances in location-specific firing after manipulations of the environment (Muller and Kubie, 1987). Nevertheless, it is also important to characterize field properties numerically. This analysis will be restricted to estimates of field sizes and of the positions of fields within the apparatus, but we realize that it is crucial to find ways of formally classifying field shapes and firing rate contours, as well as other possible properties.

Estimating the size of firing fields. In Figure 3, we can see that the size of the firing field at 7 o'clock grows with increased recording time, the field area being defined as including pixels with a firing rate greater than zero. Part of this effect is due to the undersampling of the apparatus area with short recording durations; if firing were confined perfectly to a portion of the cylinder, it would still take time for the animal to visit each pixel within the field. With longer recording times, however, the nonideal nature of spatial firing specificity becomes important. Since the firing probability outside the field is not zero, if a pixel were included in the field whenever its firing rate was greater than zero, the field area would approach the area of the cylinder as the recording time got very long.

To avoid this problem, it is convenient to define a firing field as a group of pixels that occupies a continuous part of the apparatus, where the firing rate in each pixel must be greater than some selected threshold. Pixels that do not satisfy the firing rate criterion are excluded from the field, even if surrounded by field pixels. The continuity condition is that a candidate pixel must share at least one edge with a pixel already known to be in the field; a corner is not enough (Lewis, 1977). This permits the member pixels to be found with a recursive algorithm. The minimum field size is set at 9 pixels; this avoids the absurdity of referring to fields for cells that are nearly silent (cf. Fig. $5 E$ ). In addition, only the largest field in a session is considered, in order to bypass the question of whether averages should be taken across units or across fields.

The relationship between field size and firing rate cutoff is illustrated in Figure 9; over the cutoff range, each doubling reduces average field size by about 20 pixels. Even with a threshold of $8.0 \mathrm{AP} / \mathrm{sec}$, the average size is an appreciable fraction (4\%) of the apparatus area. It therefore appears that the fields of a small number of cells (relative to the number of place cells) will blanket the apparatus area. The small number of cells needed to cover the area may depend in part on the limited size of the small cylinder, but if recording is done in larger apparatuses, the average field area grows (Muller and Kubie, 1987). Thus, 
A

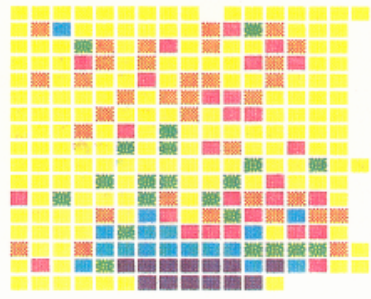

B

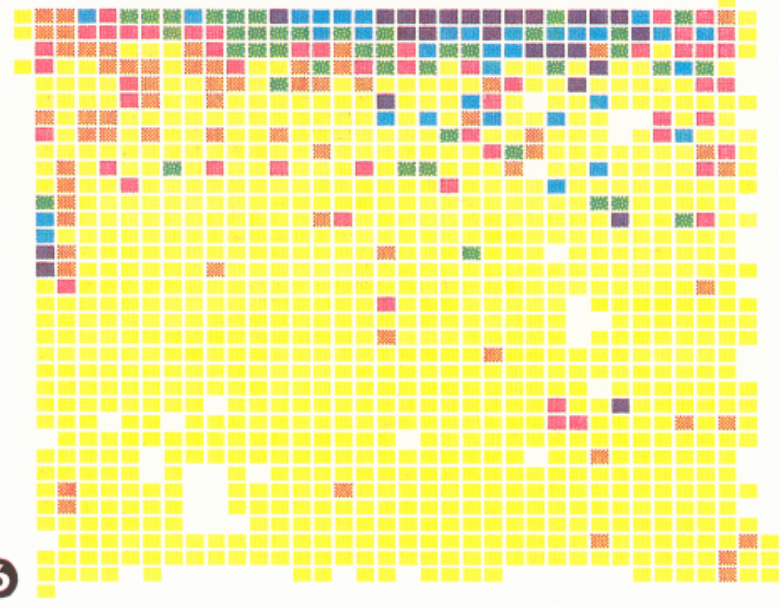

A

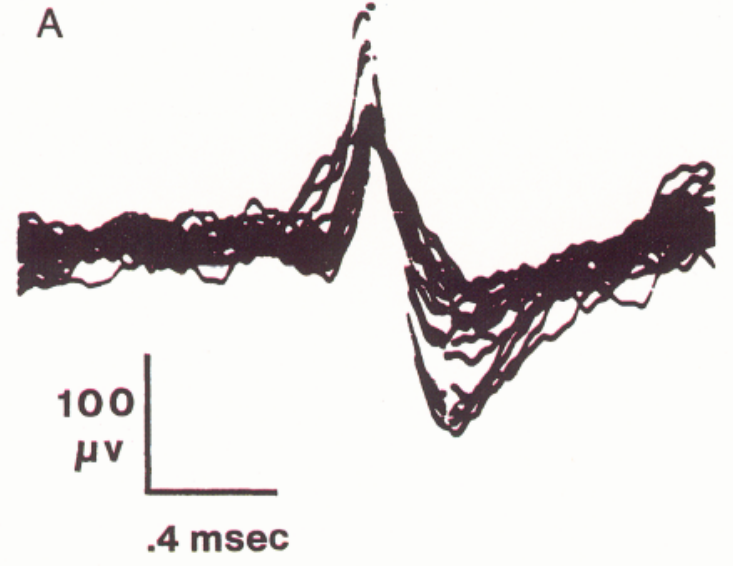

C
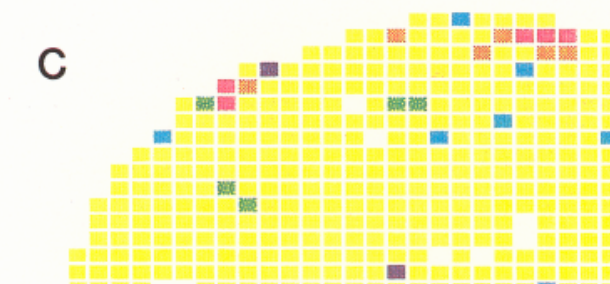

믐
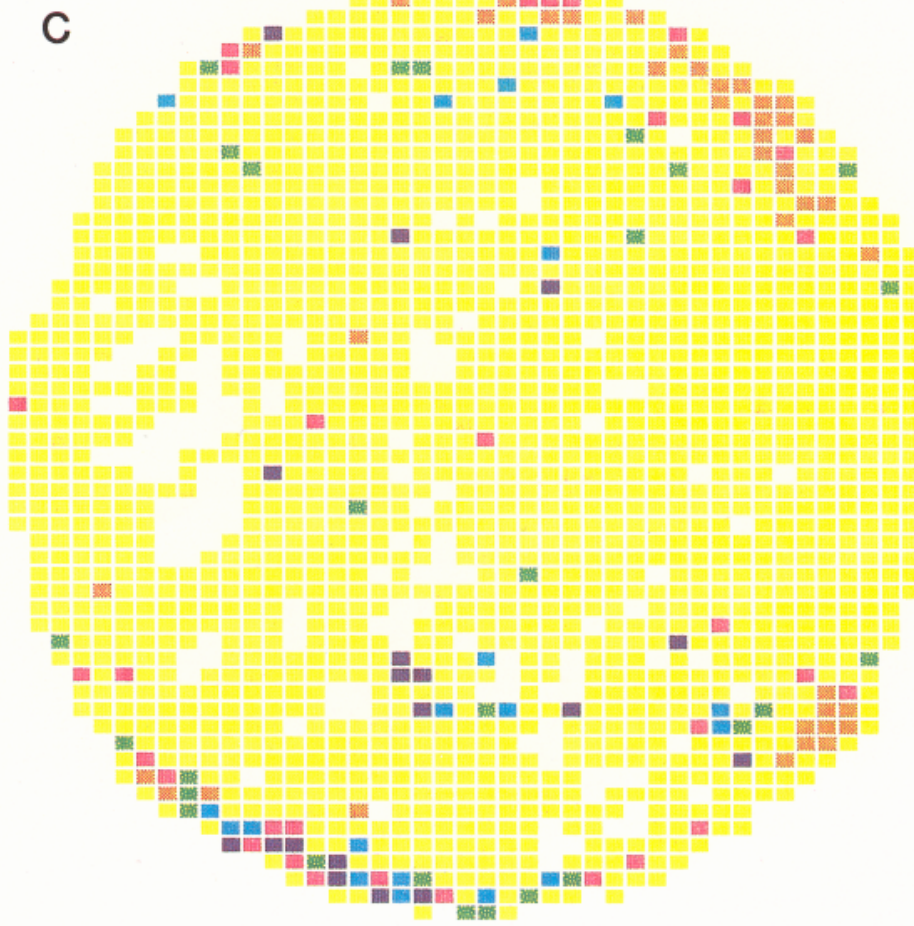
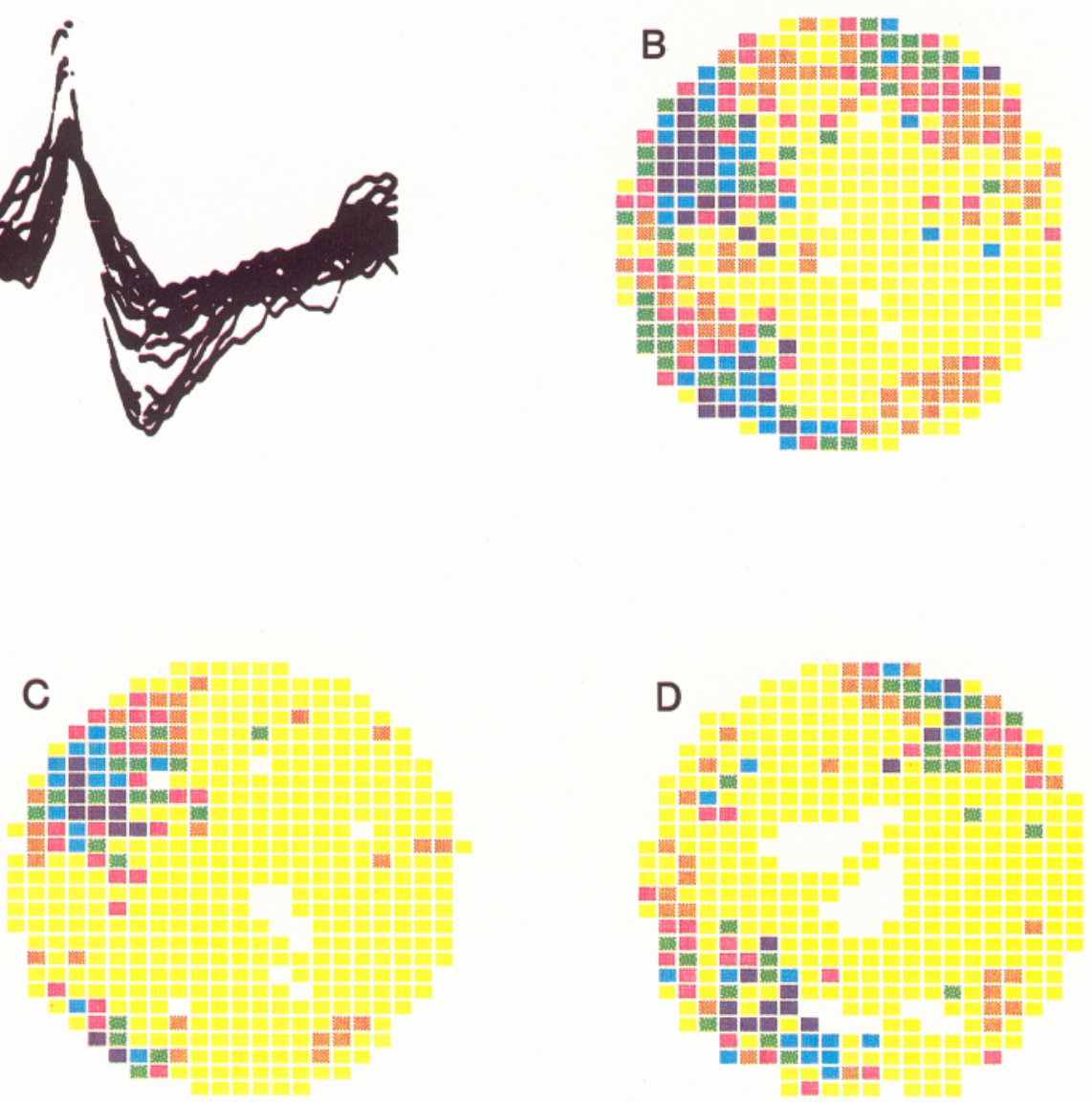

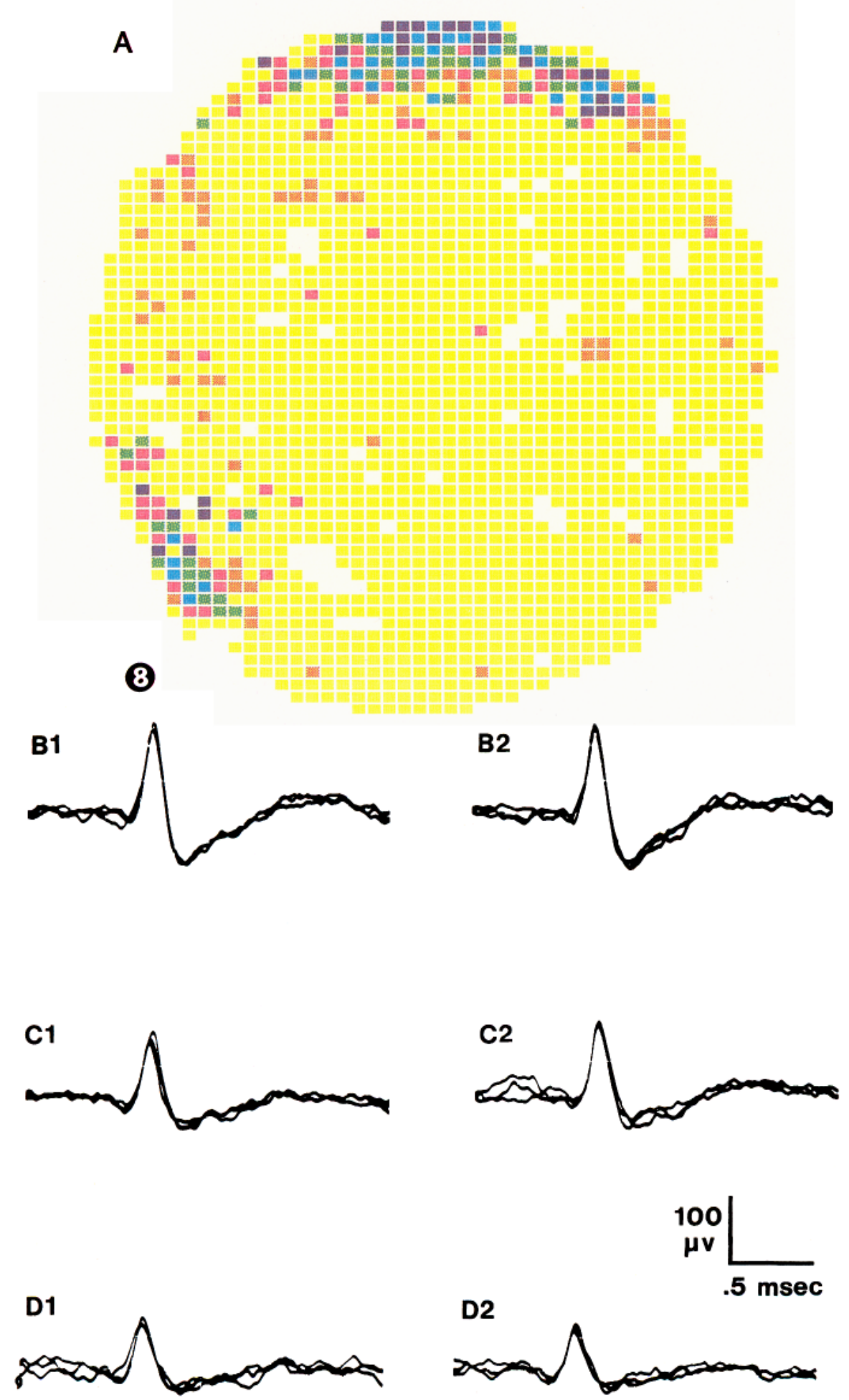

Figure 8. Rate map with 2 firing fields. This session was recorded in the large cylinder. $A$, Rate map with 1 field at 12 o'clock and another at 8 o'clock. After the session was finished and the map printed, the rat was held in the 12 o'clock field and the 3 superimposed action potentials in $B 1$ were photographed. The rat was then held in the other field and the 3 action potentials in $B 2$ were photographed. The electrode was then advanced by about 15 $\mu \mathrm{m}$, and the action potential traces in $C 1$ and $C 2$ were taken, again by holding the rat in the $12(\mathrm{Cl})$ and $8(\mathrm{C2})$ o'clock fields. The traces in $D I$ (12 o'clock) and D2 (8 o'clock) were obtained after a second $15 \mu \mathrm{m}$ electrode advance. $A$, R21S4B16; unit R21U1 (CA3/4). Median rates: $0.0 ; 1.43 ; 3.16 ; 6.34 ; 11.0$; 16.4.

Figure 6. Edge-conforming fields in the small rectangle, large rectangle, and large cylinder. $A$, Linear field in the small rectangle. $B$, Linear field in the large rectangle that runs nearly the entire length of one of the long walls. $C$, Crescentic field in the large cylinder. There is a faint, diametrically symmetric field at 2 o'clock. $A$, R2S9B16A; unit R2U2B (CA3/4). Median rates: 0.0; 0.25; 0.60; 1.24; 3.01; 10.00. B, R3S38B16; unit R3U6 (CA3/ 4). Median rates: $0.0 ; 0.90 ; 2.73 ; 4.90 ; 8.44 ; 13.9$. $C$, R8S7B16; unit R8U1 (CA1). Median rates: $0.0 ; 0.041 ; 1.15 ; 1.91 ; 3.16 ; 6.27$.

Figure 7. Discrimination of 2 action potential waveforms present on the same wire. $A$, Four superimposed traces for the larger-amplitude action potential. Also an indeterminate number $(<10)$ of traces for the smaller unit. $B$, Rate map for both units. In this session, the discriminator windows were wide open, so that action potentials from both units were accepted. There are 3 firing fields, at 1:30, 7:30, and 10 o'clock. Median rates are not given. $C$, Rate map for the larger of the 2 units. The field at 10 o'clock in $B$ is from this unit. $D$, Rate map for the smaller of the 2 units. The 2 fields at $1: 30$ and 7:30 o'clock in $B$ are from this unit. Some spillover from the nonselected cell is seen in the maps of $C$ and $D$. $C$, R7S9B8; unit R7U1C (CA1). Median rates: 0.0; 1.12; 3.02; 6.90; 12.3; 21.1. D, R7S10B16; unit R7U1B (CA1). Median rates: 0.0; $1.05 ; 2.40 ; 3.77 ; 7.5 ; 13.0$. 


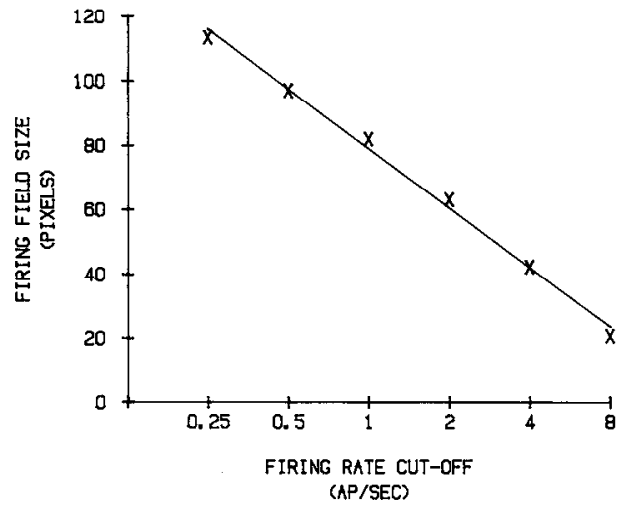

Figure 9. Firing field size as a function of firing rate cutoff. Average field size (for 34 cells) is plotted against the action potential frequency cutoff used to determine whether a pixel was counted as part of the field. Note that the frequency scale is logarithmic. The correlation between field size and $\log$ (cutoff) was -0.995 . The line is the least-squares regression line.

there seems to be a great degree of overlap of the firing fields of place cells.

In the absence of a firm basis for selecting the firing rate cutoff for inclusion of pixels in a field, we will use the value of 1.0 $\Lambda \mathrm{P} / \mathrm{sec}$ for subsequent calculations; $1.0 \mathrm{AP} / \mathrm{sec}$ is approximately the time-averaged firing rate for entire sessions (i.e., ignoring position), averaged over all cells. It is also our subjective estimate of what constitutes "significant" spatial firing. Nevertheless, we realize that a less arbitrary method for cutoff selection would be desirable.

Using the $1.0 \mathrm{AP} / \mathrm{sec}$ threshold, we can calculate the correlation between field size and maximum firing rate, as given by the rate in the median pixel for the fastest (purple) rate category. The low value ( $r=0.055$; see the scattergram in Fig. 10) indicates that maximum firing rate (range, $4.78-43.3 \mathrm{AP} / \mathrm{sec}$ ) is a poor predictor of field size (range, $200-2818 \mathrm{~cm}^{2}$ ). Low correlations between field size and maximum firing rate are also found with other values of the cutoff and another estimate of maximum firing rate (rate within the field center pixel; see below). Thus, a single parameter is not enough to characterize firing fields, even if their shape is ignored. In particular, it seems that the steepness of the firing rate gradients can vary independently of the peak rate.

Spatial distribution of fields within the apparatus. In the third part of this section, it was seen that there is no tendency of fields to occur where the animal prefers to spend its time. A related issue is whether fields are more likely to occur in a particular portion of the cylinder. This may be answered by looking at the areal distribution of field centers. The field center is defined as the pixel that has the highest firing rate when an average is taken that includes its 8 nearest neighbors; the results are not very different if the pixel with the highest rate is used. An added constraint is that the candidate for field center must have been part of the original field. This method consistently chooses centers that agree with our subjective estimates from the inspection of rate maps.

Figure 11 is a plot of the field centers of 34 cells ( 6 cells did not have fields with the rate cutoff at $1.0 \mathrm{AP} / \mathrm{sec}$; only 32 symbols appear because the centers of 2 pairs of fields coincided). For statistical analysis, the cylinder was divided into equal-area concentric rings, and the number of field centers that fell within

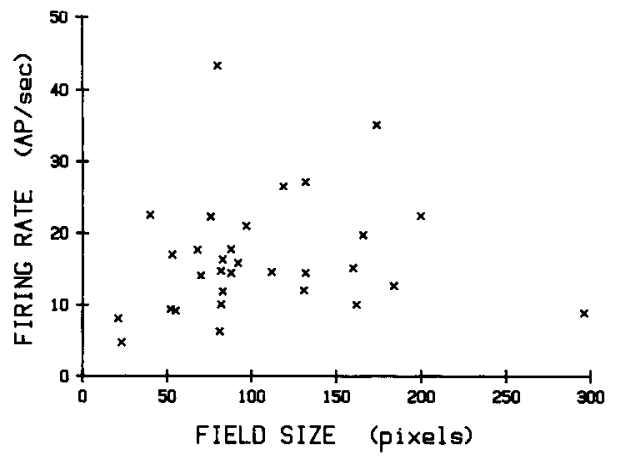

Figure 10. Scattergram of maximum firing rate as a function of field size. Maximum firing rate was taken to be the rate in the median purple (fastest firing rate category) pixel. Field size was calculated by using a threshold of $1.0 \mathrm{AP} / \mathrm{sec}$ for inclusion of a pixel in the field. The very low correlation is also seen if maximum rate is estimated from the rate in the field center.

each ring was counted. Chi-squared tests revealed no tendency for fields to occur at a certain distance from the center of the cylinder as the number of rings was varied from 2 to 6 . A similar treatment of the angular distribution of field centers showed that they have no tendency to occur in any one wedge of the cylinder when the number of wedges ranged between 2 and 6 . Thus, in the small cylinder, the probability of occurrence of field centers is everywhere the same. It is interesting that there appears to be nothing special about the region immediately adjacent to the cue card.

Can the animal's position be calculated from the firing of place cells? Perhaps the simplest interpretation of the spatial firing of place cells is that the firing rate of each cell signals the animal's distance from the field center, and that the animal's position is an average of the centers of all cells that are active (firing at more than $1 \mathrm{AP} / \mathrm{sec}$ ). This "distance" hypothesis can be numerically checked by determining, for each pixel in the apparatus, the subset of place cells whose fields include the pixel; a cell is part of the subset only if its firing rate in the pixel is $>1.0$ $\mathrm{AP} / \mathrm{sec}$. Next, the length of the vector that connects the pixel to the center of each active field is weighted by the firing rate of the cell; the weighting method is given in the legend to Figure 12. Finally, the sum of the weighted vectors is taken. The resultant vector points from the animal's assumed position to its calculated position, and is an estimate of the error in the computed position.

The distance hypothesis systematically finds the wrong position for the rat when it is applied to the place cells recorded in the small cylinder. Figure $12 \mathrm{~A}$ summarizes the $X$ component of the error in calculated position. For each pixel, an arrow was plotted in the direction of the $X$ component if the error exceeded 0.5 pixels; if the error was smaller, no arrow was plotted. The same process was carried out for the $Y$ component of the error in Figure $12 B$. The scheme works well near the center of the apparatus, where the relative error is smaller (fewer arrows per area) and where there is no bias in the direction of the errors. Near the wall, however, the arrows point towards the diameter normal to the component of the error. Thus, the calculated position is systematically wrong at the apparatus boundary. The distance hypothesis fails because it makes no provision for the effects of the apparatus boundary. Given that fields occur homogeneously in the apparatus, there must be more active cells whose fields lie between the animal and the far wall than there 


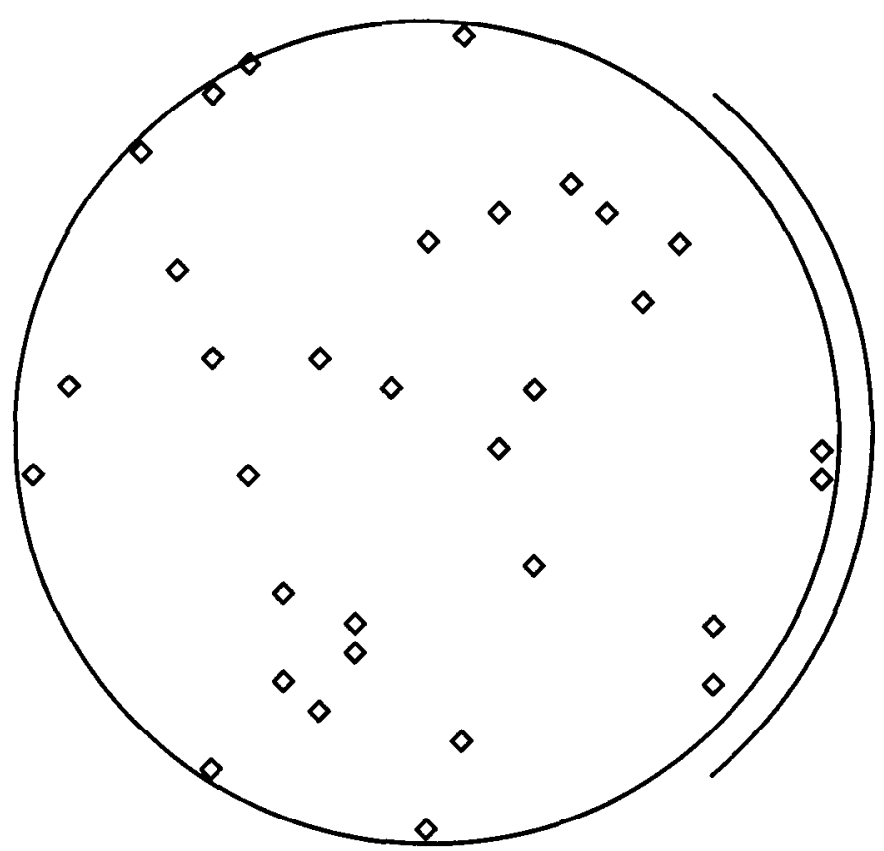

Figure 11. Positions of field centers in the small cylinder. Arc outside the cylinder represents the polarizing stimulus. The method for locating the field centers is given in the text. By eye, it appears that field centers are homngeneously distributed; this impression is confirmed by a simple statistical analysis (see text).

are between the animal and the near wall. It follows that error vectors will in general point towards the center of the cylinder.

\section{CA1 versus $C A 3 / 4$ complex-spike cells}

Since the connectivity patterns of pyramidal cells in CA1 differ from those of CA3/4, and since place cells are thought to be pyramidal cells, comparisons between the firing properties of place cells recorded from the 2 pyramidal cell regions are of interest. A chi-squared test revealed no difference in the occurrence of crescent-like versus circular or elliptical fields between CA1 $(n=11)$ and CA3/4 $(n=23)$ cells $(p=0.22)$. The average firing field area (with a $1.0 \mathrm{AP} / \mathrm{sec}$ cutoff) for CA1 cells was $22.3 \%$ of the cylinder area; the average size for $\mathrm{CA} 3 / 4$ cells was $22.0 \%$ of the cylinder area. The $t$ value for the difference in mean field size was very low $(t=0.21 ; p<0.42)$. The average maximum firing rate (estimated from the field center rate) was 15.1 for CA 1 cells and $20.9 \mathrm{AP} / \mathrm{sec}$ for CA $3 / 4$ cells. The $t$ value for the difference in the average maximum firing rates was just short of the 0.05 level of probability $(t=1.57 ; p<0.06)$. Virtually the same result was obtained $(t=1.48 ; p=0.07)$ when the sample was made to include an additional 22 cells, 12 of which were from CA1. It is therefore possible that CA3/4 place cells fire at somewhat higher rates than do place cells in the CA1 region.

\section{Discussion}

A major purpose of the experiments described in this paper was to demonstrate that the spatial firing of place cells is measurable with objective, automatic methods. The strength of the phenomenon is directly visible in the color-coded firing rate maps that were used to summarize the spatial firing distributions. From such maps, it is seen that place cell activity is well described as occurring in "firing fields" whose locations are tightly bound to specific portions of the apparatus and are stable in
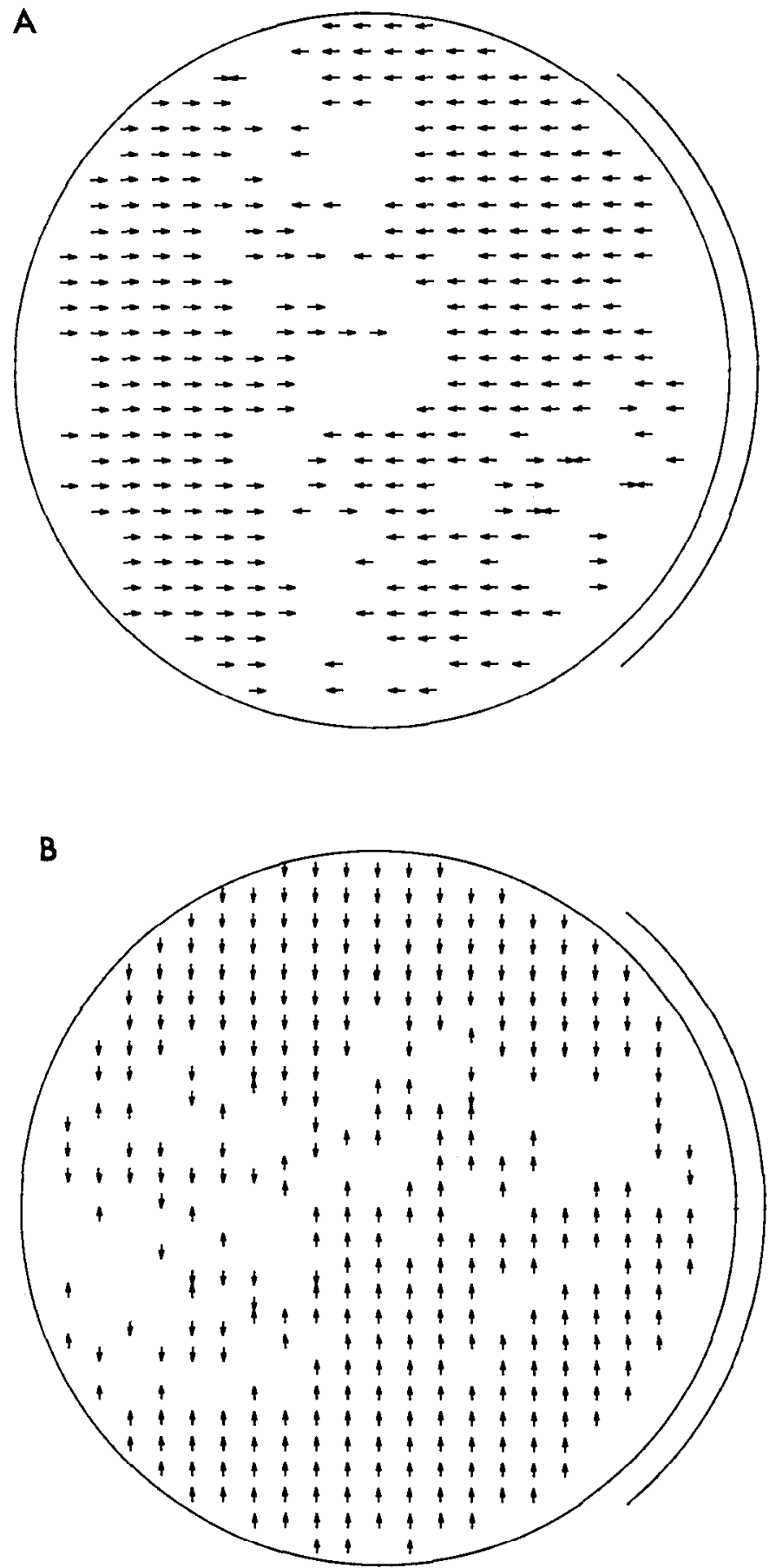

Figure 12. Plots of the $X(A)$ and $Y(B)$ components of the error vector that points from the animal's assumed position to the position calculated from the firing of the set of place cells whose fields include the assumed position. For each place cell whose field included the pixel, the length of the vector from the assumed position to the field center was weighted by the relative firing rate of the cell in the assumed position. The vector length was multiplied by 0.2 if its firing rate was in the lowest rate category, 0.4 if its rate was in the second rate category, and so on. Thus, if the pixel was in the fifth (highest) rate category, the vector length was unchanged. An arrow is plotted for a pixel if the appropriate component of the error was greater than 0.5 pixels; the direction of the arrow indicates the direction of the error. The pattern of arrows indicates that the relative size of the error is smaller in the apparatus center than near its wall. From the $X$ and $Y$ components of the error vector, it is seen that it generally points towards the center of the cylinder. Arcs in $A$ and $B$ represent the position of the polarizing stimulus. 
time. The contrast between in-field and out-of-field firing is high, and the firing rate gradients within fields are steep. It is important to point out that robust firing fields were seen, even though the animal was not encouraged to treat subregions of the apparatus differently from one another. The significance of this phenomenon is bolstered by the fact that a large fraction of wellisolated complex-spike cells appear to act as place cells; it is our impression that place cells comprise upwards of $60 \%$ of complex-spike cells.

\section{Location-specific versus behavior-specific correlates of place cell firing}

Two explanations of the observed spatial firing patterns are available. On the one hand, it is possible that the firing of each place cell is correlated with a certain behavioral state of the animal, and that firing fields arise because each state happens to occur only when the animal is in a particular part of the apparatus. This behavior-specific explanation stands in contrast to the possibility that the proper correlate of place cell firing is the animal's position within the apparatus, in which case the firing would be location-specific. One method of distinguishing between these possibilities would be to train rats to walk constantly at the same speed and to change direction randomly; walking is clearly the desirable behavior, since it is important that the firing of the cell be measured everywhere in the apparatus. If firing ficlds wcrc obscrved under these circumstances, it could no longer be maintained that behavior-specificity was necessary and sufficient to account for the spatial firing.

The behavior exhibited by the rat in these experiments only approximates a random walk; thus, additional considerations are needed to reject the behavior-specific notion. One deviation from the ideal behavior is that some of the animal's time is spent eating food pellets. This is not, however, a serious objection because the fraction of time the animal eats is less than $10 \%$ of the total time, and because eating itself is homogeneously distributed over the area of the apparatus. In addition, direct observation reveals no strong correlation between firing and eating or other, even less frequent, activities. Our data are not sufficiently complete for us to deny that place cells sometimes fire in association with certain behaviors, but this effect is at most second order in the current experiments.

A second argument in favor of location-specificity comes from the demonstration that the firing rate of place cells is, on the average, uncorrelated with the spatial distribution of dwell time within a recording session. As would be expected if place cell firing and an animal's propensity to spend more time in parts of the environment were independent, the correlation between firing and dwell time was positive for some cells, negative for others, and indistinguishablc from zcro for most cells. This argument is reinforced by the observation that firing fields are homogeneously distributed within the apparatus, despite the fact that rats tend to spend more of their time near the cylinder wall. The final argument in favor of the location-specific interpretation of place cell firing comes from the observation that fields recorded while the animal moves freely can be reproduced when the animal is carried around in the apparatus by hand ( $\mathrm{J}$. L. Kubie and R. U. Muller, unpublished observations). This dissociation of firing and behavior is at odds with the pure behavior-specific hypothesis of place cell activity.

As noted, the current results do not imply that place cell firing must be independent of behavior. In fact, there is a strong indication that the behavioral state may be correlated with spatial firing. Kubie et al. $(1984,1985)$ have shown that place cell firing covaries with the state of the hippocampal EEG. In rats tested in the current task, the infield firing rate was higher and the out-of-field rate was lower during theta $(6-8 \mathrm{~Hz}$ sine-like activity) than during other EEG states. Note that the same fields were seen during non-theta EEG states, although they were weaker, with a higher background rate. Since the hippocampal EEG state is highly correlated with the animal's behavioral state (Vanderwolf, 1969), it is likely that place cell firing would be found to be correlated with behavior. In particular, since walking is the predominant theta-correlated behavior in these experiments, it is very likely that the ratio of in-field to out-of-field firing is higher during walking than otherwise. Thus, behavior may modulate the overall spatial firing pattern. It is also possible that circumstances can be found in which correlates of behavioral firing greatly outweigh spatial correlates for a majority of complex-spike cells. Nevertheless, the results show that there are conditions in which place cell firing is strongly locationspecific.

\section{What is signaled by the firing of place cells?}

A straightforward interpretation of place cell activity is that firing rate signals the rat's distance from the cell's field center. A single cell cannot carry enough information to locate the animal accurately, but the simultaneous firing of many place cells could signal position as accurately as necessary. This "distance" hypothesis is attractive because of its simplicity, but several arguments suggest that it needs serious modification.

First, any place cells that have 2 fields will act as noise sources in the determination of the animal's location. If cells with 2 fields exist, they are in the minority, and it is possible that 1 -field cells can accurately estimate the animal's position; however, other interpretations should be examined before dismissing the significance of 2-field cells. One interpretation is that 2 (or more) "maps" of the environment may coexist within the hippocampus. In several papers, it has been shown that single cells often exhibit unrelated spatial firing in each of 2 (or more) apparatuses (O'Keefe and Conway, 1978; Kubie and Ranck, 1983; Muller and Kubie, 1987), which suggests that the hippocampus can map the accessible space differently at different times. It may be that the small cylinder is not treated as a unit by the hippocampus, and that 2 -field cells indicate that the apparatus is broken up into subregions. Certainly, this possibility would arise if the environment consisted of 2 rooms connected by a doorway, and a single cell had a field in each room. The multiplemap idea has the virtue of internal consistency; since an animal can only be in one place at a time, it eliminates the need to think about second fields as noise. A different interpretation of 2 -field cells is that their activity represents a special relationship between the 2 regions of the apparatus in which the cell fires fastest. For instance, the 2 firing fields might be the endpoints of frequently used trajectories through the apparatus. In any case, it is clear that 2-field cells should not be dismissed as noise until much more is known about the hippocampal "mapping" system.

A second finding that bears on the message carricd by placc cells is that firing fields come in a variety of shapes. The idea that a cell's firing rate signals the rat's distance from the field center can be supported only for circular fields, or for circular fields that are truncated by an impenetratable barrier. For other shapes, each firing rate is associated with a range of distances from the field center, and firing rate does not encode distance 
unambiguously. Even cells with circular fields in the cylinder are not necessarily signaling distance; radially symmetric fields may simply be one of the field shapes that develop in circular environments.

The most direct argument against the distance hypothesis comes from the simulation in which the animal's calculated position was compared to its assumed position for each pixel in the apparatus. The simulation was systematically in error whenever the animal was assumed to be near the apparatus boundary because of the lack of fields on the other side of the wall. One way of bringing the results of the simulation into line with the spatial theory of O'Keefe and Nadel (1978) is to postulate that the mapping system is non-euclidean in nature.

We conclude that the idea that each place cell signals the animal's distance from a point is suspect. It is important to realize, however, that this conclusion is not in conflict with the basic notion that the hippocampus is involved in processing spatial information; it only means that the significance of place cell firing is not as obvious as it first seems.

\section{Limitations of the analysis of place cells}

The only behavioral variable measured in the present experiments was the animal's position on the floor of the small cylinder. There are, however, a number of other factors that might modulate place cell firing. As suggested above, it is possible that factors such as walking speed or direction, along with position, influence firing. McNaughton et al. (1983a) found that in-field firing is somewhat faster if the animal's running speed is higher. The 4 studies that looked at the relationship between orientation and place cell activity came to different conclusions. O'Keefe and Dostrovsky's (1971) seminal paper on place cells paid more attention to orientation than to position as the key firing correlate. A later publication by O'Keefe (1976) stated that few cells showed orientation sensitivity. Olton et al. (1978) reported that none of their units showed differential firing when the rat moved in versus out on arms of an 8 -arm maze. Most recently, McNaughton et al. (1983a) found that most cells in their sample were directionally selective under conditions very similar to those used by Olton et al. (1978). In the present work, we observed that orientation was at most a second-order factor for place cell firing. In particular, it was noted that the firing of cells with edge fields was about the same when the animal moved along the cylinder wall in either direction. Nevertheless, it is clear that the determination of place cell firing by behavioral factors other than position requires much more study.

A different limitation concerns the tracking of lights on the rat's head. There are 2 problems with the implicit assumption that it is reasonable to treat the rat as a point. First, it is not clear how to pick the optimal placement for the lights. If the same session were analyzed with lights on the rat's neck or tail, the spatial firing distribution would be altered. The head seems like the natural body part to associate with the position of a point rat, but even different head placements would lead to somewhat different results. Neglecting the rat's body is also misleading, since it gives rise to the notion that the rat can do anything anywhere in the apparatus. This is incorrect, in that the motions and behaviors that can be performed near the cylinder wall are different than those possible in open space. Thus, a full analysis of place cell firing must consider mechanical, as well as neural, factors.

The third major limitation in the current treatment is the lack of attention paid to the action potential time series generated by place cells. This issue gains importance directly from the observation that, occasionally, a place cell with a well-defined firing field is silent when the rat passes near or through the field center. Such strong departures from location-specific firing will have to be explained before we can claim that place cell activity is properly understood.

We therefore consider the data presented in this paper as a first step in the analysis of place cell firing. The limitations cited above will have to be removed, but we will show in the following paper (Muller and Kubie, 1987) that the techniques of data collection and reduction introduced here are sufficient to explore the essential question of environmental control over place cells and their firing fields.

\section{References}

Berger, T. W., P. C. Rinaldi, D. J. Weisz, and R. F. Thompson (1983) Single unit analysis of different hippocampal cell types during classical conditioning of rabbit nictitating membrane response. J. Neurophysiol. 50: 1197-1219.

Best, P. J., and J. B. Ranck, Jr. (1982) The reliability of the relationship between hippocampal unit activity and sensory-behavioral events in the rat. Exp. Neurol. 75: 652-664.

Best, P. J., and L. T. Thompson (1984) Hippocampal cells which have place field activity also show changes in activity during classical conditioning. Soc. Neurosci. Abstr. 10: 125.

Fox, S. E., and J. B. Ranck, Jr. (1975) Localization and anatomatical identification of theta and complex-spike cells in the dorsal hippocampal formation of rats. Exp. Neurol. 49: 299-313.

Fox, S. E., and J. B. Ranck, Jr. (1981) Electrophysiological characteristics of hippocampal complex-spike and theta cells. Exp. Brain Res. 41: 399-410.

Fox, S. E., S. Wolfson, and J. B. Ranck, Jr. (1986) Hippocampal theta rhythm and the firing of neurons in walking and urethane anesthetized rats. Exp. Brain Res. 62: 495-508.

Kubie, J. L. (1984) A driveable bundle of microwires for collecting single-unit data from freely moving rats. Physiol. Behav. 32: 115118.

Kubie, J. L., and J. B. Ranck, Jr. (1983) Sensory-behavioral correlates in individual hippocampal neurons in three situations: Space and context. In Neurobiology of the Hippocampus, W. Seifert, ed., pp. 433-447, Academic, New York.

Kubie, J. L., R. U. Muller, and J. B. Ranck, Jr. (1983) Manipulations of the geometry of environmental enclosures control the spatial firing of hippocampal neurons. Soc. Neurosci. Abstr. 9: 646.

Kubie, J. L., S. E. Fox, and R. U. Muller (1984) Variations in place cell firing with the state of the hippocampal EEG. Soc. Neurosci. Abstr. 10: 599.

Kubie, J. L., R. U. Muller, and S. E. Fox (1985) Firing fields of hippocampal place cells: Interim report. In Electrical Activity of the Archicortex, G. Buzsaki and C. H. Vanderwolf, eds., Akademia Kiado, Budapest.

Lewis, P. (1977) Maps and Statistics, Methuen, London.

McNaughton, B. L., C. A. Barnes, and J. O'Keefe (1983a) The contributions of position, direction and velocity to single unit activity in the hippocampus of freely moving rats. Exp. Brain Res. 52: 41-49.

McNaughton, B. L., J. O'Keefe, and C. A. Barnes (1983b) The stereotrode: A new technique for simultaneous isolation of several single units in the central nervous system from multiple unit records. J. Neurosci. Methods 8: 391-397.

Muller, R. U., and J. L. Kubie (1987) The effects of changes in the environment on the spatial firing of hippocampal complex-spike cells. J. Neurosci. 7: 1951-1968.

Muller, R. U., J. L. Kubie, and J. B. Ranck, Jr. (1983) High resolution mapping of the spatial fields of hippocampal neurons in the freely moving rat. Soc. Neurosci. Abstr. 9: 646 .

O'Keefe, J. (1976) Place units in the hippocampus of the freely moving rat. Exp. Neurol. 51: 78-109.

O'Keefe, J. (1979) A review of the hippocampal place cells. Prog. Neurobiol. 13: 419-439.

O'Keefe, J. (1983) Spatial memory within and without the hippocampal system. In Neurobiology of the Hippocampus, W. Seifert, ed., pp. 375-403, Academic, New York. 
O'Keefe, J., and D. H. Conway (1978) Hippocampal place units in the freely moving rat: Why they fire where they fire. Exp. Brain Res. 31: 573-590.

O'Keefe, J., and J. Dostrovsky (1971) The hippocampus as a spatial map. Preliminary evidence from unit activity in the freely-moving rat. Brain Res. 34: 171-175.

O'Keefe, J., and L. Nadel (1978) The Hippocampus as a Cognitive Map, Clarendon, Oxford, UK.

Olton, D., M. Branch, and P. Best (1978) Spatial correlates of hip- pocampal unit activity. Exp. Neurol. 58: 387-409.

Pellegrino, L. S., A. S. Pellegrino, and A. J. Cushman (1967) A Stereotaxic Atlas of the Rat Brain. Plenum, New York.

Ranck, J. B., Jr. (1973) Studies on single neurons in dorsal hippocampal formation and septum in unrestrained rats. Exp. Neurol. 41:461555.

Vanderwolf, C. H. (1969) Hippocampal electrical activity and voluntary movement in the rat. Electroencephalogr. Clin. Neurophysiol. 26: $407-418$. 